\title{
The linear perspective information in ground surface representation and distance judgment
}

\author{
Bing Wu ANd ZiJiang J. He \\ University of Louisville, Louisville, Kentucky \\ AND \\ Teng Leng OOI \\ Pennsylvania College of Optometry, Elkins Park, Pennsylvania
}

\begin{abstract}
Most ground surfaces contain various types of texture gradient information that serve as depth cues for space perception. We investigated how linear perspective, a type of texture gradient information on the ground, affects judged absolute distance and eye level. Phosphorescent elements were used to display linear perspective information on the floor in an otherwise dark room. We found that observers were remarkably receptive to such information. Changing the configuration of the linear perspective information from parallel to converging resulted in relatively larger judged distances and lower judged eye levels. These findings support the proposals that (1) the visual system has a bias for representing an image of converging lines as one of parallel lines on a downward-slanting surface and (2) the convergence point of a converging-lines image represents the eye level. Finally, we found that the visual system may be less sensitive to the manipulation of compression gradient information than of linear perspective information.
\end{abstract}

A number of empirical studies have shown that the ground surface substantially influences object localization in the intermediate distance range $(2-25 \mathrm{~m})$, supporting the ground theory of space perception advocated by J. J. Gibson $(1950,1979)$. For instance, distance judgment is accurate when a target on the ground is seen with reference to a homogeneous ground surface (Feria, Braunstein, \& Andersen, 2003; He, Wu, Ooi, Yarbrough, \& Wu, 2004; Loomis, Da Silva, Fujita, \& Fukusima, 1992; Loomis, Da Silva, Philbeck, \& Fukusima, 1996; Sinai, Ooi, \& He, 1998; B. Wu, He, \& Ooi, 2007). The visual system is also able to use the ground surface as a reference for localizing an object that does not directly have physical contact with the ground surface (Madison, Thompson, Kersten, Shirley, \& Smits, 2001; Meng \& Sedgwick, 2001, 2002; Ni, Braunstein, \& Andersen, 2004; Ooi \& He, 2006; J. Wu, $\mathrm{He}, \&$ Ooi, 2004). All of these results, however, do not preclude the idea that the visual system can use other types of visual environmental information than the ground surface for space perception (see, e.g., Lappin, Shelton, \& Rieser, 2006; J. Wu, He, \& Ooi, 2003). Nevertheless, it has been shown, for example, that observers are more efficient at processing information on a ground-like rather than a ceiling-like surface (Bian, Braunstein, \& Andersen, 2005; McCarley \& He, 2000, 2001). In an effort to understand how the visual system represents the ground surface for use as a reference frame in object localization, we have proposed a sequential surface integration process (SSIP) hypothesis (He et al., 2004; B. Wu, Ooi, \& He,
2004). The SSIP hypothesis attempts to elucidate how the ground surface representation is constructed and what visual cues in the physical space are used. Briefly, we claim that the visual system begins by constructing the near ground surface representation using reliable near depth cues (such as, e.g., vergence, accommodation, binocular disparity, and motion perspective). This near ground surface representation is then used by the visual system as a template to sequentially integrate the far surface patches, with the aid of texture gradient information, in order to form a global ground surface representation. Our goal in the present study is to investigate how the visual system utilizes texture gradient information for ground surface representation.

The ground surface often contains several types of texture gradient information, including information on linear perspective, compression gradient, texture gradient of size, and texture gradient of density (Gibson, 1950; Purdy, 1960; Sedgwick, 1986). Each of these types plays a role in contributing to depth/space perception. The linear perspective information, for instance, has been found to be an effective monocular depth cue for human space perception (see, e.g., Blessing, Landauer, \& Coltheart, 1967; Cutting \& Vishton, 1995; Gibson, 1950; Sedgwick, 1983, 1986; Vogel \& Teghtsoonian, 1972). As illustrated in Figure 1A, linear perspective lines on the ground surface that appear to converge as they run from the lower to the upper visual field convey the visual impression of parallel lines extending in depth from the near to the far surface and vanishing

Z.J.He, zjhe@louisville.edu 
A

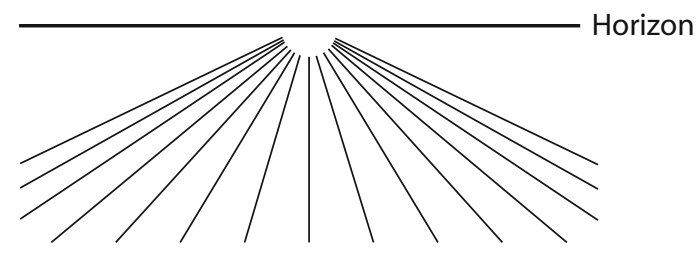

B Two parallel lines on a flat ground surface $\tan (\beta / 2)=(W \sin \alpha) /(2 H)$

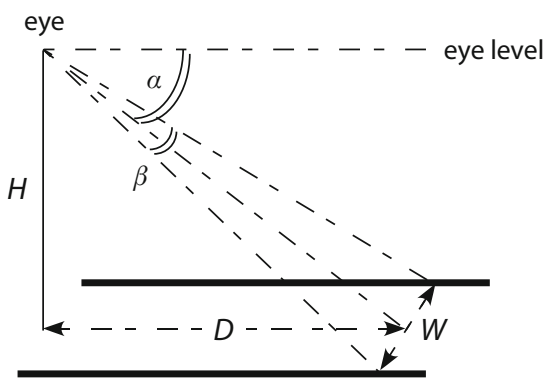

C Two converging lines slanted upward eye

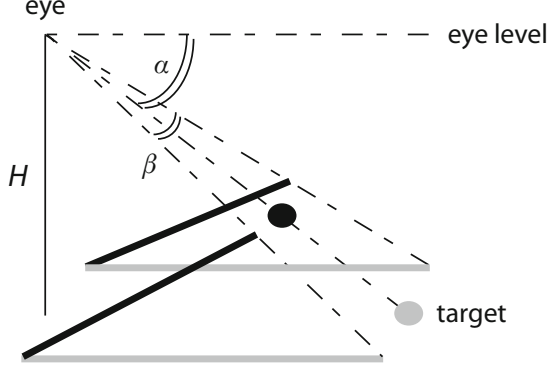

Figure 1. (A) An illustrative linear perspective drawing of a ground surface, in which parallel lines on the ground appear to converge as they extend from the near surface to the far surface, toward the horizon. (B) The retinal image of two parallel lines on a level ground surface can be described by the geometrical relationship $\tan (\beta / 2)=(W \sin \alpha) /(2 H)$. (C) The retinal image of a pair of converging lines that slant upward (black lines) is the same as the one formed by a pair of parallel lines on the level ground surface (gray lines). Therefore, both pairs of lines can be described by the same geometrical relationship.

at the horizon. In fact, such a visual interpretation of linear perspective information is the basis of two basic principles of perspective drawing in art and architecture (Vredeman de Vries, 1604/1968). The first principle states that all lines drawn to represent parallel lines in the physical space converge at a vanishing point, and the second that the vanishing point of the converging lines is located on a point on the horizon when the observer looks straight ahead. Armed with these two basic principles, artists, architects, and lay people alike can visually recover the actual spatial layouts from perspective drawings. Namely, converging lines correspond to parallel lines (the parallel-line rule), and the height of the vanishing or convergence point of the converging lines marks the height of the horizon (the horizon rule). Below, we elaborate on how the visual system can use these two rules to represent converging lines for space perception in the intermediate distance range $(2-25 \mathrm{~m})$.

The parallel-line rule assumes that the visual system has a tendency to represent the retinal image of converging lines as parallel lines. For simplicity, consider Figure 1B, in which an upright observer views a pair of parallel lines on the level ground surface. The retinal image of the two parallel lines can be described by the geometrical relationship $\tan (\beta / 2)=(W \sin \alpha) /(2 H)$, where $\beta$ is the angular width between the two lines that subtend an angular declination below the horizon of $\alpha, W$ is the linear width between the two lines, and $H$ is the observer's eye height. But this relationship aside, recovering the orientation of the lines from the retinal image could be shrouded with uncertainty. This is because there are multiple lines in the physical space that could produce the same retinal image as the two parallel lines. For example, in Figure 1C, the retinal image of the gray parallel lines on the level floor surface is the same as the retinal image of the two black converging lines that slant upward from the floor. To resolve this ambiguity in representing the lines, the visual system needs either additional depth information or internal rules that are based on assumptions of the physical space. We suggest that the parallel-line rule, which is the basis of the linear perspective depth cue, is one such internal rule. If the parallel-line rule is fully implemented, the visual system will be able to represent the retinal image of converging lines as belonging to parallel lines in the physical space.

By extension, according to the parallel-line rule, the visual system will represent a pair of converging lines on a level floor surface (Figure 2A, left) as a pair of parallel lines on a surface with a geographical slant of $\theta$ (Figure 2A, right). Such a scenario can be described by the geometrical relationship $\tan \theta=[2 H \tan (\Phi / 2)] / W_{\mathrm{s}}$, where $W_{\mathrm{s}}$ is the linear width of the two parallel lines and $\Phi$ is the angle between the two converging lines on the level floor surface.

The foregoing analysis notwithstanding, does the visual system actually use the parallel-line rule as an intrinsic guide to resolve the ambiguous retinal image? A number of empirical studies have found that observers perceive converging lines presented on the frontoparallel plane as if they were located on a slant surface. Significantly, the perceived slant of the surface is close to, but not the same as, the orientation of the surface on which the (converging) lines would be parallel (see, e.g., Braunstein \& Payne, 1969; Cutting \& Millard, 1984; Gillam, 1970; Rosinski \& Levine, 1976). This indicates that the visual system does observe the parallel-line rule, but not fully.

The horizon rule assumes that the vanishing/convergence point of converging lines is located on the horizon. Applying the horizon rule can affect the perceived eye level, which in turn affects space perception in the intermediate distance. The eye level is defined as the projection line from the eye that is parallel to the level ground surface and meets the horizon at infinity. In theory, the visual system can obtain the eye level by referring to the horizon in the natural environment, but the natural horizon is not always accessible, because of poor visibility or occlusion by obstacles. Some have suggested that the visual system can 


\section{Converging vs. Parallel Lines}

Two converging lines on a flat ground surface $\beta_{\mathrm{f}}=2 \arctan \left[W_{\mathrm{f}} \sin \alpha /(2 H)\right]$

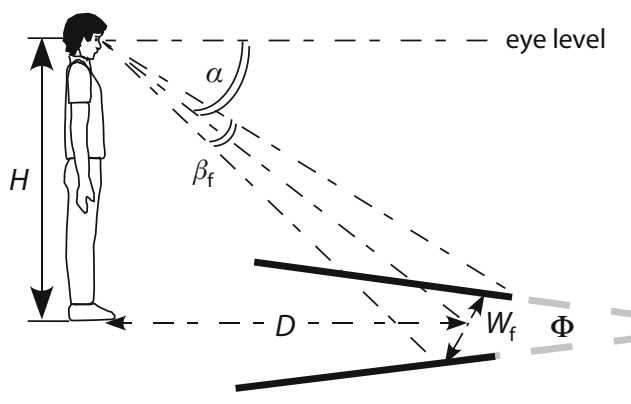

B

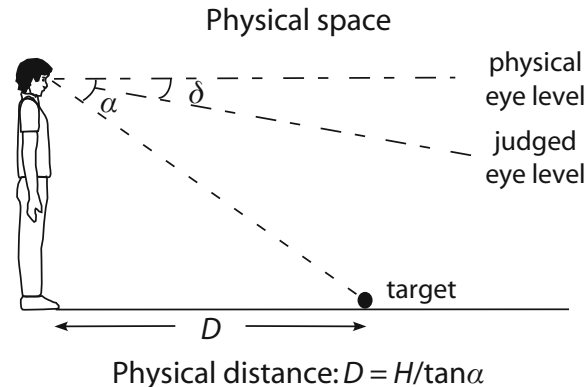

Two parallel lines on downward-slanting ground

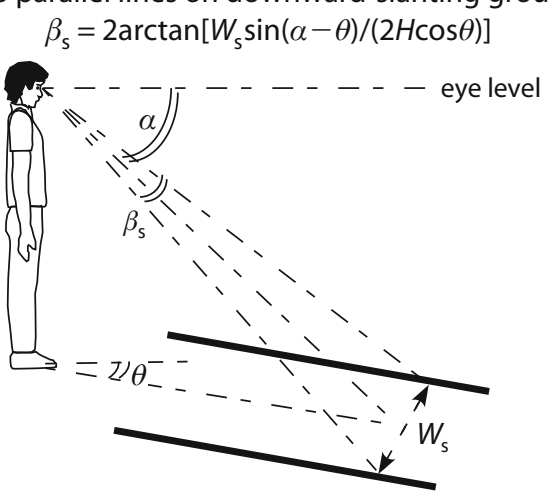

Prediction

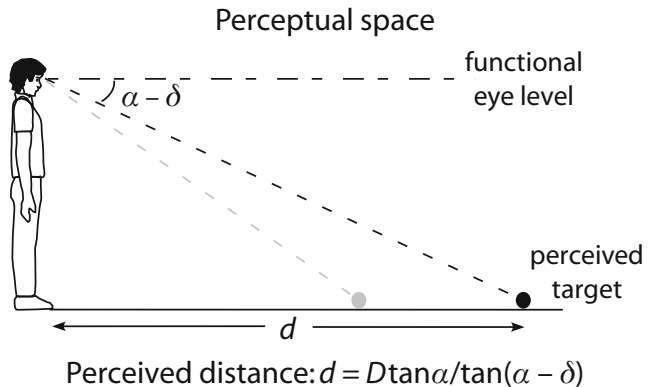

Figure 2. (A) An illustration of the implementation of the parallel-line rule to represent a pair of converging lines on a level floor. The converging lines on the floor (left) produce the same retinal image as a pair of parallel lines on a floor with a geographical slant of $\theta$ (right; $\tan \theta=[2 H \tan (\Phi / 2)] / W_{\mathrm{s}}$. Thus, if the visual system uses the parallel-line rule, it will represent the retinal image of the converging lines (on the left figure) as the parallel lines (on the right figure). (B) The left figure depicts a side view of an observer viewing a target on a level floor surface with converging lines as the background in the physical space. Using the horizon rule, the convergence point of the lines will be used as an indication of the judged eye level, and thus the judged eye level will be shifted downward relative to the physical eye level. In the perceptual space (right figure), the lowered judged eye level becomes the functional eye level used as a reference to define the angular declination of the target on the level floor. Consequently, the perceived angular declination of the target $(\alpha-\delta)$ will be smaller than its physical angular declination $(\alpha)$, and the judged distance of the target is overestimated.

reconstruct the horizon by extrapolating converging lines in the physical space to locate their vanishing/convergence point (Gibson, 1979; Sedgwick, 1973, 1986; J. Wu et al., 2003). J. Wu et al. (2003) showed that an observer's visually perceived eye level is influenced by the orientation of a pair of parallel lines. In their experiment, the observer stood on a level floor surface between two parallel phosphorescent lines that were, respectively, pasted on the left and the right side of the vertical walls in a dark hallway. The two parallel lines were located at about the observer's eye height, and their length ran from near to far in the sagittal direction. When the orientation of the lines was rotated such that their front ends were tilted downward, the judged eye level was found to shift downward. This finding suggests that the visual system can apply the horizon rule to influence the perceived eye level. However, the perceived eye level in the experiment did not shift as much as the angular tilt of the parallel lines. A likely explanation for this is that besides visual information, nonvisual information such as the body's sense of gravity can affect the perceived eye level (Bringoux, Tamura, Faldon, Gresty, \& Bronstein, 2004; Cohen, 1973; Matin \& Li, 1995).
From the preceding discussion, one could predict that when a pair of parallel lines on the level floor surface is replaced by a pair of converging lines, the perceived eye level shifts downward, and the distance of a target on the floor is overestimated. This is because, as shown on the left side of Figure 2B, the perceived eye level is a reference for angular declination (Ooi, Wu, \& He, 2001). Therefore, when the perceived eye level shifts downward $(\delta)$, the angular declination of the target on the floor is reduced from $\alpha$ to $\alpha-\delta$. Thus, in the perceptual space where the perceived eye level is treated as a line perpendicular to the observer's vertical axis (i.e., gravity axis), the angular declination of the perceived target becomes $\alpha-\delta$ (Figure $2 \mathrm{~B}$, right). Since the perceived distance is specified by the geometrical relationship $H / \tan (\alpha-\delta)$ rather than $H / \tan \alpha$, the perceived distance is overestimated.

The analysis above of converging lines on a level floor surface (Figures 1 and 2), as an example, provides the theoretical basis for our investigation into how the visual system constructs the ground surface representation using linear perspective information. We recognize that our analysis and its quantitative predictions (Figure 2) rest 
on the assumption that only linear perspective information is used for ground surface representation. Clearly, this assumption is likely to be violated in a real-world environment, since it is almost impossible to eliminate the contributions of other depth cues to ground surface representation. Nonetheless, the theoretical analysis provides us with an insight into how linear perspective information is processed by the visual system to form a ground surface representation. In order to study the role of linear perspective, in the present study we placed phosphorescent stars or lines on the level floor of a dark room. The phosphorescent elements were arranged in various configurations (e.g., parallel or converging lines) that allowed us to manipulate linear perspective information while excluding or minimizing other types of texture gradient information on the ground. Upon such ground surface backgrounds, and depending on the experimental goals, our observers responded to their perception by performing either a distance judgment task or an eye level judgment task.

Experiments 1-3 demonstrate that linear perspective information contributes to distance judgment and, by extension, to ground surface representation. Experiment 4 reveals that the human visual system is quite efficient at using linear perspective information for representing the ground surface. Experiment 5 shows that, besides influencing the observers' perceptual responses (verbal reports of perceived distances), linear perspective information can influence responses in a visually directed task (blind throwing). Experiment 6 illustrates that converging lines on a level floor surface cause the judged eye level to shift downward and an overestimation of distance (Figure 2B). Experiment 7 examines the impact on distance perception of compression gradient information on the ground surface. Compression gradient information is a texture gradient cue that manifests as an incremental reduction in the sagittal visual angles (i.e., in the depth) of equally spaced horizontal lines from near to far. We found that the visual system is less effective at extracting the compression gradient information for representing a ground surface than it is at extracting the linear perspective information.

\section{EXPERIMENT 1 Full Cue Versus Linear Perspective Cue in the Dark}

The visual system relies on its intrinsic bias to determine the location of a dimly lit target when the ground surface is not visible in the dark (see, e.g., Ooi, Wu, \& He, 2001, 2006; B. Wu et al., 2004; J. Wu, He, \& Ooi, 2005). The intrinsic bias is an implicit curve surface that extends from the observer's feet into the distance; the local slant of this surface (upward and away from the floor) increases with distance. (For simplicity, in the present article we will assume that the implicit surface has a constant slant.) The implicit surface causes the distance of a dimly lit target on the level ground surface to be underestimated in the dark (Ooi et al., 2001, 2006; Philbeck \& Loomis, 1997; B. Wu et al., 2004; J. Wu et al., 2005).

Recently, using an exocentric task, a separate study in our laboratory furnished further evidence for the notions that the intrinsic bias takes the form of an implicit slant surface and contributes to ground surface representation (J. Wu, He, \& Ooi, 2006). In that study, two conditions were tested in the dark. In the first, an L-shaped target was placed on the level floor, which was not visible in the dark. In the second, the level floor surface was delineated with six phosphorescent elements arranged in two parallel rows, upon which the L-shaped target was placed. The observer judged the orientation of the L-shaped target by gesturing with his or her hand to indicate the perceived slant of the target. We found that (1) the observers judged the target slant to be larger in total darkness (Condition 1) than in the presence of the texture background (Condition 2), and (2) the judged slant increased with distance. We suggest that the slant in total darkness revealed the slant of the intrinsic bias, whereas the slant with the limited texture background revealed the weighted contributions of the intrinsic bias and the external depth cues to ground surface representation (Ooi \& He, 2007; Ooi et al., 2006; B. Wu et al., 2007; J. Wu et al., 2004). In a separate study that used computer visual displays incorporating photographed grass fields of the full-cue environment, Sauer, Braunstein, Andersen, and Bian (2001) also suggested that an L-shaped target on the ground was perceived as slanted upward and that the slant increased with distance.

When more reliable depth information is available on the ground, however, the relative contribution of the intrinsic bias is reduced, and the ground surface representation becomes closer to veridical. For example, the foreshortening of an L-shaped target on the level ground surface of a fullcue environment is less than the foreshortening in a dark environment. This finding suggests that the perceived slant of the L-shaped target and that of the ground surface representation is smaller in a full than in a dark environment (Ooi et al., 2006; B. Wu et al., 2004). Furthermore, using the blind-walking paradigm, a number of studies have produced findings that egocentric distance judgment is accurate on a homogeneous ground surface in a lit, full-cue environment (Creem-Regehr, Willemsen, Gooch, \& Thompson, 2005; Elliott, 1987; Loomis et al., 1992; Loomis et al., 1996; Ooi et al., 2001; Rieser, Ashmead, Talor, \& Youngquist, 1990; Sinai et al., 1998; Thomson, 1983; B. Wu et al., 2004). Presumably, this is because the homogeneous ground surface, which carries abundant depth information, is accurately represented in the light (He et al., 2004).

In the present experiment, we presented the linear perspective cue on the floor of a dark room in order to measure judged egocentric distance. To produce the linear perspective cue, we arranged small, star-shaped phosphorescent elements in parallel rows on the floor. We predicted that, since linear perspective is an effective source of depth information, distance judgment in an otherwise dark environment would be quite accurate.

For the sake of simplicity in describing our stimulus design in the remainder of this article, we will refer, when appropriate, to the ground texture formed by small, starshaped phosphorescent elements as dot texture. Similarly, we will refer to ground texture formed by phosphorescent stripes as line texture. 


\section{Method}

Observers. Eight observers participated in the experiment after providing informed consent. They all had normal or corrected-tonormal visual acuity and were naive to the purpose of the experiment. In this and in subsequent experiments, the observers viewed all displays with both eyes.

Design. The two independent variables were texture background and target distance $(1.5,2.5,3.75,5.0$, and $6.25 \mathrm{~m})$. The texture background on the level floor was presented in two conditions: (1) a test condition, with a dot-texture array presented in the dark, and (2) a baseline condition, with a full-cue environment in the light. The observers were first presented with the test condition and then with the baseline condition.

Stimuli. The test condition was presented in a dark room $3 \times$ $11 \mathrm{~m}$ in size. The dot texture comprised a $3 \times 9$ array of green starshaped phosphorescent plastic elements placed on a level, carpeted floor. The overall dimensions of each star-shaped phosphorescent element were $2.54 \times 2.54 \mathrm{~cm}$, with a $1-\mathrm{mm}$ thickness. The phosphorescent elements in the dot-texture array were arranged $0.6 \mathrm{~m}$ apart horizontally and $1.0 \mathrm{~m}$ apart sagittally (in depth; see Figure 3).

The glow intensity of the phosphorescent elements was sufficiently high for observers to see the elements, but not for them to see the floor and the surrounding environment. (This criterion for the illumination level of the phosphorescent elements was also used in subsequent experiments.) In addition to the dot-texture array, two star-shaped phosphorescent elements were placed (laterally) by the observer's feet where he or she stood during the experiment (the origin). The horizontal separation of the two elements was $1.0 \mathrm{~m}$, and the observers were informed that they could use this distance as a reference for judging the distance to the test target during the experiment. The target used in the test condition was an internally illuminated red ping-pong ball $\left(0.16 \mathrm{~cd} / \mathrm{m}^{2}\right)$. The ball was encased in a contraption with an adjustable iris-diaphragm aperture that was set at an angular size of $0.23^{\circ}$ when viewed at eye level for all the distances tested.
The baseline condition was conducted in the same room with the light turned on. The test target was an orange-colored hockey puck $7.62 \mathrm{~cm}$ in diameter and $2.54 \mathrm{~cm}$ in height. For both the conditions, the order of testing the five target distances was randomized. Each target distance $(1.5,2.5,3.75,5.0$, or $6.25 \mathrm{~m})$ was tested twice.

Procedure. A verbal report task (see, e.g., Philbeck \& Loomis, 1997) was employed for obtaining the observer's judgment of the absolute distance of the target on the floor. Before the experiment, each observer was given a practice session to familiarize him- or herself with the verbal report task. The practice session, which used a verbal report procedure similar to the one in the test session (see below), was performed outside the test room in a lighted hallway. The target here was a hockey puck placed at a number of random distances between 0.6 and $12 \mathrm{~m}$. The observers were not provided any feedback on the accuracy of their performance.

When an observer was ready for the experiment, he or she was led into a small, lighted waiting room adjacent to the test room. The waiting room was essentially used as a "transit room" for the observers between trials, to prevent them from observing preparation of the stimulus displays. As a further precaution, the view of the display was blocked from the observer by a curtain that hung near the "origin" observation point. To prepare for a trial, the experimenter informed the observer to switch off the waiting room's light and to walk into the test room. The observers were guided by two small arrays of phosphorescent star-shaped elements on the floor, toward which they were asked to walk. Upon reaching the star arrays, the observer stepped onto the stars and fully covered them with the left and right shoes; doing this positioned the observer at the origin point. When the observer verbally informed the experimenter of being ready for the trial, the dimly lit red test target was turned on at a predetermined test distance, and the curtain that blocked the observer's view of the stimulus was removed. (This preparation procedure was used for all of the experiments reported in this article except for Experiment 5.)
Test Condition

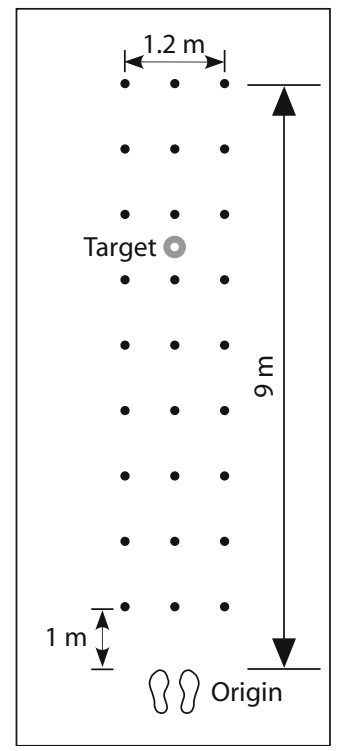

\section{Experiment 1}

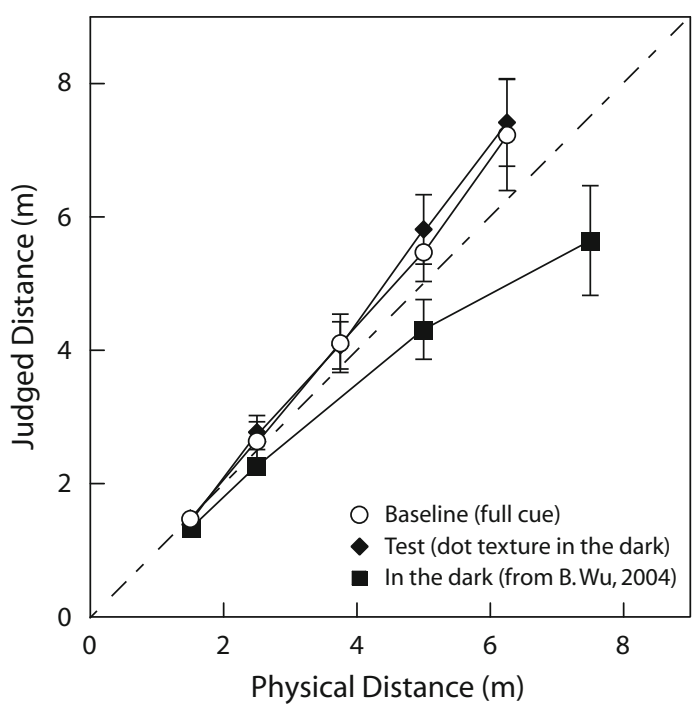

Figure 3. Experiment 1. (Left) The ground surface layout in the test condition, in which small phosphorescent elements (dot texture) were placed on the level floor to provide linear perspective information in an otherwise dark room. (Right) Judged target distance as a function of physical distance. Performance in the test condition (diamonds) was similar to that in the baseline (full-cue; open circles) condition. These data were compared with the judged distances (square symbols) from a previous experiment in the dark, which were underestimated. 
In this experiment and in all the later ones we will report, the observers were explicitly informed that the test target and texture patterns were on the level floor surface. Observers viewed the scene with both eyes and estimated the distance of the target from their feet. Except in Experiment 5 (the blind-throwing task), they then verbally reported a numerical estimate of the distance, either in feet and inches or meters and centimeters. After making the verbal response, they walked back to the waiting room. Meanwhile, the experimenter repositioned the curtain to block the view of the test area and prepared the test target for the following trial. No feedback was provided to observers regarding their performance. A similar experimental procedure was used in the baseline condition.

\section{Results}

The open circles and filled diamonds of the graph in Figure 3 represent the average reported distances in the baseline and test conditions, respectively. Clearly, these two sets of data are very similar to each other [two-way ANOVAs with repeated measures: main effect, $F(1,7)=1.190$, $p>.1$; interaction, $F(4,28)=2.258, p>.08$ ]. If one assumes that the visual system can accurately represent the ground surface in the full-cue condition, our findings suggest that the dot-texture background in the test condition also carried sufficient information to construct an accurate ground surface representation.

For an informal comparison, we have also plotted in Figure 3 a set of verbal report data (black squares) from a separate distance judgment experiment performed in complete darkness with no visual cues. These data were obtained in a previous study with a different group of observers in our laboratory (B. Wu, 2004). Clearly, these data reveal that distance is underestimated in the dark when the ground surface is not visible (Ooi et al., 2001, 2006; Philbeck \& Loomis, 1997; B. Wu et al., 2004; J. Wu et al., 2005).

\section{EXPERIMENT 2 Convergence Versus Parallel Conditions}

Experiment 1 suggested that an array of regularly spaced dot-texture pattern as the background in an otherwise dark environment carries sufficient depth information to allow participants to judge distances as accurately as in a full-cue environment. In Experiment 2, we further manipulated the spacing of the dot-texture pattern in two conditions in order to assess how linear perspective information is utilized. These are the convergence and paralleltexture conditions shown in Figure 4. According to the prediction described in the introduction and depicted in Figure 2B, the distance of a target on the level ground surface should be judged to be farther in the convergence condition than in the parallel condition.

\section{Method}

Observers. After providing informed consent, 12 observers participated in this experiment, 4 of whom had participated in Experiment 1 . All observers had normal or corrected-to-normal visual acuity and were naive to the purpose of the experiment.

Design. Two independent variables were used in a cross-factorial design. The independent variables were the texture background (convergence vs. parallel) and target distance $(1.5,2.5,3.75,5.0$, and $6.25 \mathrm{~m}$ ). Each observer performed 10 trials, which were blocked according to the texture background (convergence vs. parallel). The testing order of the two blocks was counterbalanced across observers. Furthermore, the test order of the trials within each block was fully randomized.

Stimuli. Except for the use of the texture backgrounds shown in Figure 4, the experimental setting was identical to the test condition in Experiment 1. The background in both the convergence and parallel conditions comprised a $3 \times 4$ dot-texture array. The distance of the near edge of the texture background from the observer in each condition was $1.0 \mathrm{~m}$ (Figure 4). The same dimly lit, red test target used in Experiment 1 was used in this experiment.

Procedure. The procedure was the same one used in Experiment 1.

\section{Results}

The mean reported distances are plotted in the graph in Figure 4 . These data were subjected to a two-way $(2 \times 5)$ repeated measures ANOVA with texture background (convergence vs. parallel) and target distance as factors. Overall, the results and analysis are consistent with the

Experiment 2
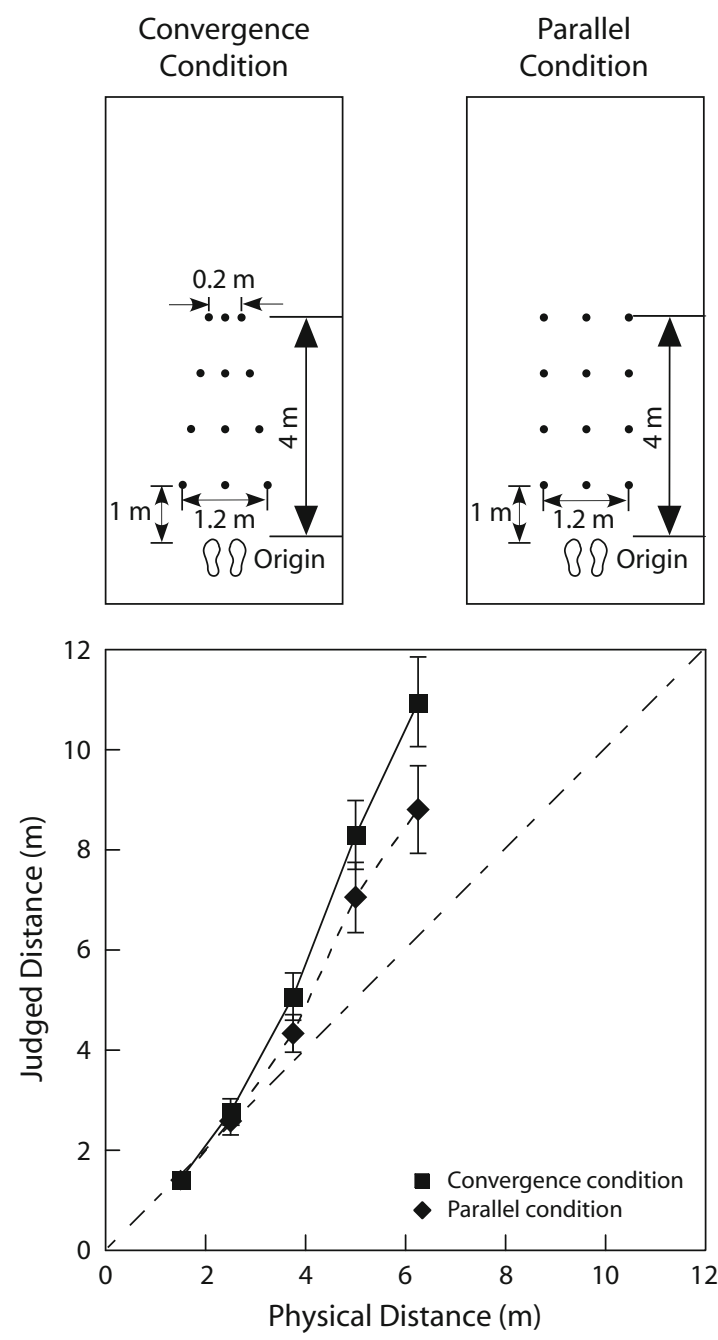

Figure 4. Experiment 2. (Top) Ground surface layouts in the convergence and parallel conditions, using dot-texture displays. (Bottom) Judged target distance as a function of physical distance. Relative to those in the parallel condition (diamonds), judged distances in the convergence condition (squares) were larger. 
prediction that observers would judge distance to be farther in the convergence than in the parallel condition. This tendency increases with target distance [main effect, $F(1,11)=6.557, p<.03$; interaction, $F(4,44)=59.231$, $p<.001]$.

One aspect of these results surprised us: the overestimation of distance in the parallel condition, which is somewhat at odds with common expectation. Most studies, including our own (J. Wu et al., 2004, 2006), have found a tendency to underestimate distance in the dark or in reduced-cue conditions. We believe that the distance overestimation in the present experiment is largely due to the fact that our observers were explicitly told that both the target and phosphorescent elements that constituted the texture background were placed on the floor. Armed with this knowledge, our observers probably overcompensated for the tendency to perceive distances as nearer in the dark. (We will defer further discussion of this issue until the General Discussion section.)

According to the model depicted in Figure 2 (the parallel-line rule), we would expect the ground surface representation to have a downward slant $(\theta)$ of $-19.2^{\circ}$ in the convergence condition (the negative value indicates a downward shift). And following the horizon rule, the same amount of shift would be expected in the perceived eye level $(\delta)$. To evaluate this prediction, we used the leastsquares criterion to fit the distance data from each of the two conditions (Figure 4) to obtain values for the empirical eye level shift. These values were $-4.4^{\circ}$ and $-6.4^{\circ}$ for the parallel and convergence conditions, respectively. The difference, $-2^{\circ}$, which reflects the impact of false linear perspective information of the convergence lines, is clearly much smaller than the predicted shift of $-19.2^{\circ}$.

The smaller-than-expected impact of the false perspective of the converging lines on either the ground surface representation or perceived eye level is likely related to the fact that the texture elements on the floor also provided other depth cues, besides linear perspective, that contributed to the ground surface representation. These cues could include texture gradient of size and density, compression gradient, relative binocular disparity gradient (since the observers viewed with both eyes), and motion parallax (owing to minute movements by the observers, even though they were instructed to minimize head/body movements during the trials). These other depth cues were present both in the parallel and convergence conditions in our experiment, even as we attempted to reveal the impact of linear perspective on ground surface representation by manipulating the linear perspective information. Therefore, the obtained slant difference cannot be attributed solely to the false linear perspective information; it must also have resulted from the interaction between the false linear perspective cue and other depth cues. For example, since binocular viewing was used in the present study, the relative binocular disparity information of the texture elements had a weighted contribution to ground surface representation. In the convergence condition, the relative binocular disparity gradient correctly specified a horizontal (level) slant, whereas the false linear perspective information specified a downward slant in ground surface representation. Accordingly, both types of depth cues could contribute to the resultant surface slant, and thus the ground surface representation would be less slanted than that specified by the false linear perspective information alone (and predicted by the parallel-line rule).

We mentioned in the introduction that studies of perceived surface slants using converging lines in the frontoparallel plane have revealed that the visual system utilizes the linear perspective cue, but not fully (e.g., Braunstein \& Payne, 1969; Cutting \& Millard, 1984; Gillam, 1970; He \& Ooi, 2000; Rosinski \& Levine, 1976). Clearly, although our study used a different empirical approach, we are reminded of the similar observations by others.

\section{EXPERIMENT 3 \\ Line-Texture Versus Dot-Texture Information}

With dot-texture backgrounds composed of small, starshaped phosphorescent elements, Experiment 2 revealed greater distance overestimation in the convergence condition than in the parallel condition. In the present experiment, we used line-texture backgrounds (phosphorescent stripes, depicted in Figure 5B) to reveal the effect of texture patterns (convergence vs. parallel) on distance judgment. There are two reasons for testing with line texture. First, whereas line texture carries only linear perspective information, dot texture carries linear perspective as well as compression gradient information. Furthermore, the compression gradient information remains the same in both the convergence and parallel conditions in the dottexture displays (see Figure 5A), giving rise to a confounding factor that might have diluted the effect of linear perspective information, particularly in the convergence condition. However, this confounding factor might not be an issue if linear perspective information had a larger weighted contribution to ground surface representation.

The second reason for conducting the present experiment using line rather than dot texture is a possible enhancement of the linear perspective "signal" with the former texture. One could argue that prior to extracting linear perspective information from a dot-texture display, the visual system needs to integrate or group variously aligned dots into distinct perceptual "line units." Perhaps the process of such an integration (of dot texture) leads to a weaker linear perspective signal in comparison with that obtained directly from a line texture. Consequently, a weakened linear perspective cue signal could have reduced the difference we found between the convergence and parallel conditions in Experiment 2. For these reasons, the present experiment compared the difference between distance judgments in the parallel and convergence conditions using line-texture as well as dot-texture backgrounds.

\section{Method}

Observers. After providing informed consent, 8 observers participated in this experiment, 2 of whom had participated in Experiment 1 . All observers had normal or corrected-to-normal visual acuity and were naive to the purpose of the experiment. 


\section{Experiment 3}
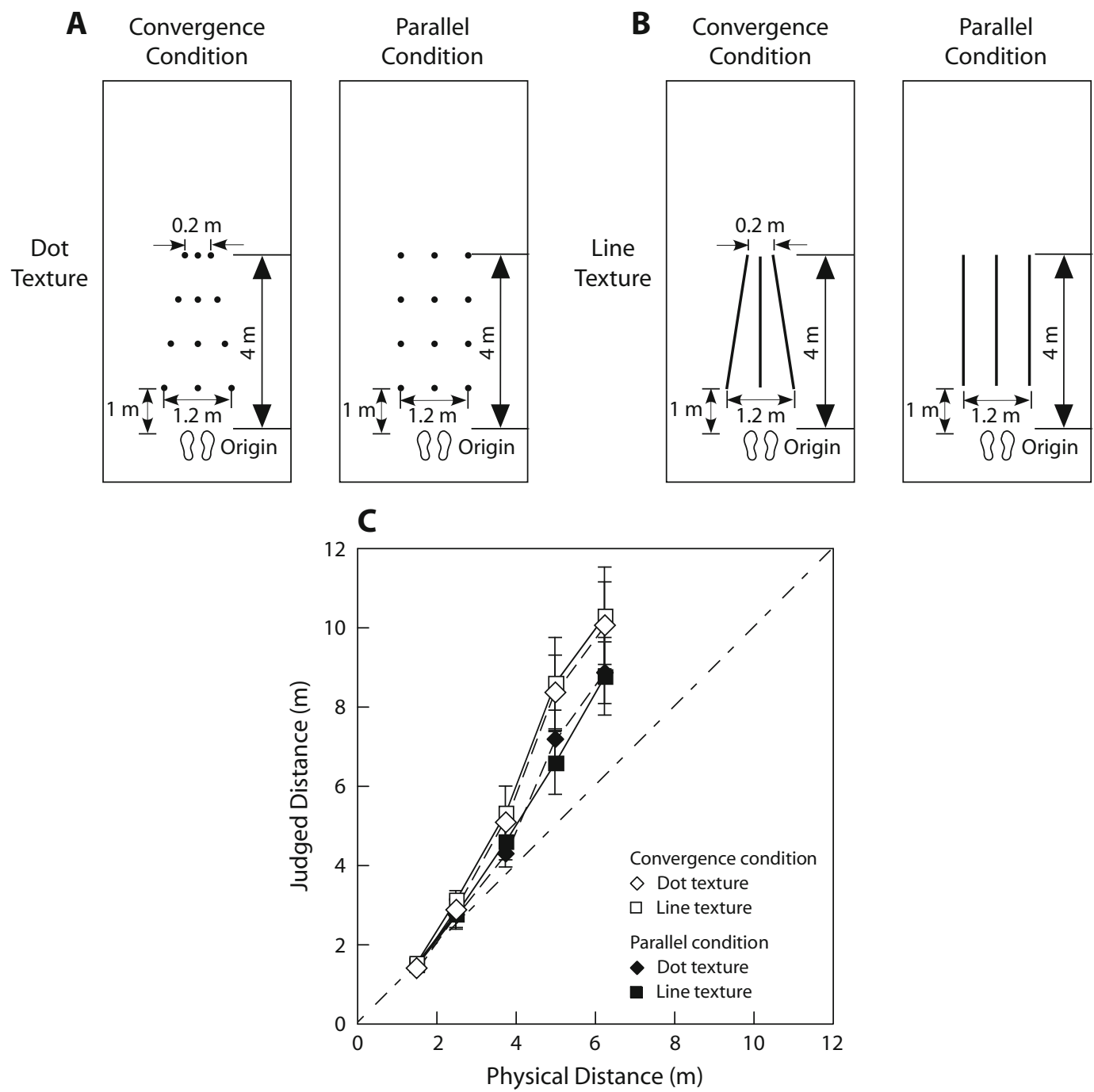

Figure 5. Experiment 3. (A and B) Ground surface layouts in the convergence and parallel conditions using dottexture displays (A) and line-texture displays (B). (C) Judged target distance as a function of physical distance. The judged distances with both the dot-texture and line-texture displays were larger in the convergence condition (open symbols) than in the parallel condition (filled symbols).

Stimuli. The $3 \times 4$ dot-texture array backgrounds (Figure 5A) were the same used in the two texture conditions (convergence and parallel) in Experiment 2. The line-texture backgrounds for the convergence and parallel conditions consisted of three phosphorescent green lines in each condition (Figure 5B). The dimensions of each phosphorescent line were $2.54 \mathrm{~cm} \times 3 \mathrm{~m}$, with 1 -mm thickness. The test target was the same one used in the test condition of Experiment 1 (a dimly lit red light $0.23^{\circ}$ in size).

Design and Procedure. Three independent variables were used, in a $2 \times 2 \times 5$ design. These variables were the texture background (parallel vs. convergence), texture element type (dot texture vs. line texture), and target distance $(1.5,2.5,3.75,5.0$, and $6.25 \mathrm{~m})$. A total of 20 trials, grouped into four blocks according to the texture patterns shown in Figures 5A and 5B, were run for each observer. Each block comprised 5 randomly shuffled trials at different target distances. The testing order of the four blocks was counterbalanced across observers. The same experimental procedure was employed as in Experiment 1.

\section{Results and Discussion}

The graph in Figure 5 plots the mean reported distances. These data were subjected to a $2 \times 2 \times 5$ repeated measures ANOVA. The results and analyses showed that, as in Experiment 2 , observers overestimated distance more in the convergence condition than in the parallel condition, and that this tendency increased with the target distance [main effect of texture background, $F(1,7)=12.927, p<.01$; texture background $\times$ distance, $F(4,28)=4.689, p<.005$ ].

Furthermore, the data reveal that judged distances were not significantly different between the dot-texture (diamonds) and line-texture (squares) backgrounds [main effect of element type, $F(1,7)=0.394, p>.5$; element type $\times$ texture background, $F(1,7)=3.403$, $p>.1$; element type $\times$ distance, $F(4,28)=0.699, p>$ 
.5 ; three-way interaction, $F(4,28)=1.383, p>.25]$. This finding suggests that, in comparison with the data from the line-texture (Figure 5B) background conditions, the additional compression gradient information that was present in the dot-texture background conditions (Figure 5A) did not substantially contribute to the data on judged distance.

There is an alternative, though more complex, explanation for our finding of no difference between the dot-texture and line-texture conditions. It is possible that the effect of eliminating the compression gradient information in the line-texture background (Figure 5B) was compensated for by stronger linear perspective information. (That is, the lines of the line texture made the linear perspective information more salient.) Admittedly, the present experiment cannot rule out this alternative explanation; nevertheless, our original explanation is also consistent with the results of Experiment 7 (below).

\section{EXPERIMENT 4 Minimum Information for Linear Perspective}

How effective is the visual system at extracting and utilizing linear perspective information for making distance judgments? To investigate this question, we next reduced the number of star-shaped phosphorescent elements making up a dot-texture display to four elements (Figure 6). We chose this number because four is the minimum number of elements required to produce linear perspective.

\section{Method}

Observers. Eight new observers participated in the experiment after providing informed consent. All had normal or correctedto-normal visual acuity and were naive to the purpose of the experiment.

Stimuli. The experimental settings are shown in Figures 6A and 6B. The four elements of the dot-texture backgrounds were either located with the nearest dots $1.0 \mathrm{~m}$ from the observer (Figure 6A) or $2.5 \mathrm{~m}$ from the observer (Figure $6 \mathrm{~B}$ ). For each texture location,
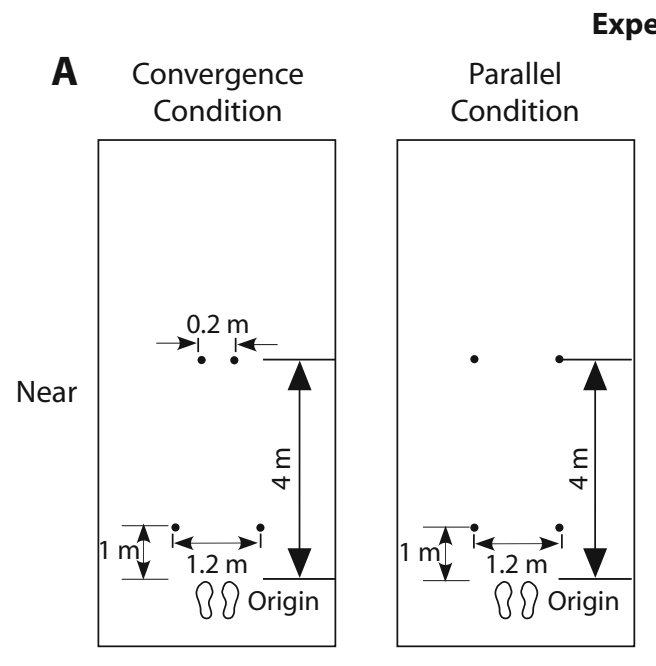

\section{Experiment 4}

B

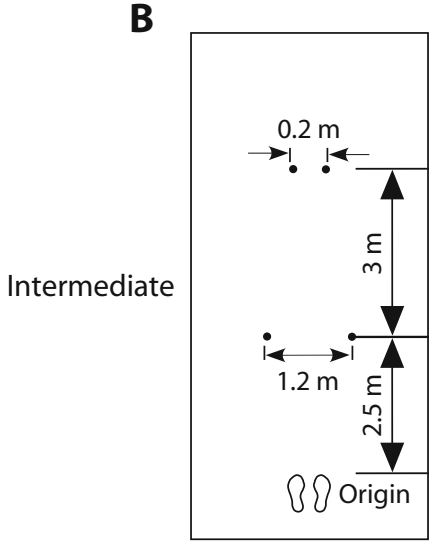

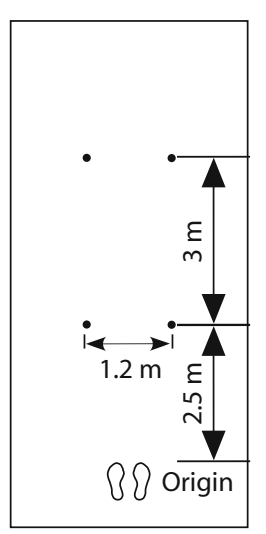
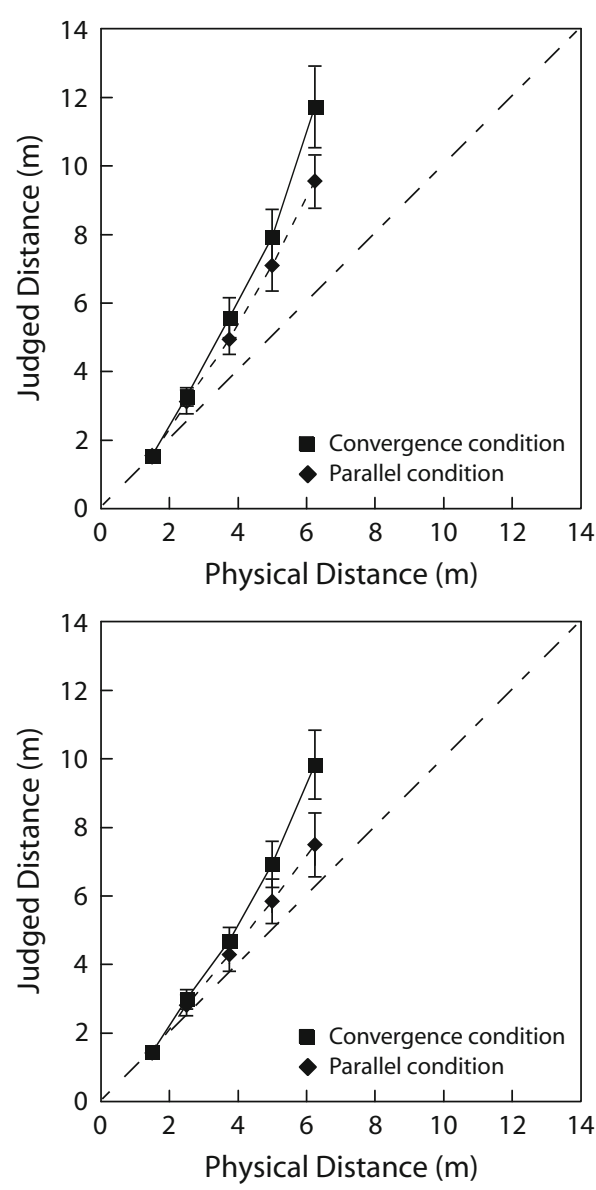

Figure 6. Experiment 4. (Left) Ground surface layouts in the convergence and parallel conditions using just four phosphorescent elements to provide linear perspective information. The dot texture was located either near (A) or at an intermediate distance from (B) the observer. (Right) The graphs in rows A and B plot judged target distance as a function of physical distance in the near and intermediate texture location conditions, respectively. Overall, the judged distances were larger in the convergence condition (squares) than in the parallel condition (diamonds). 
the parallel and convergence conditions were tested. The same dimly lit, red test target employed in Experiment 1 was used in this experiment.

Design and Procedure. Three independent variables were used in a cross-factorial design with two repeated measures. The independent variables were texture background (convergence vs. parallel), texture location (near vs. intermediate), and target distance $(1.5,2.5,3.75,5.0$, and $6.25 \mathrm{~m})$. Each observer performed 40 trials, which were blocked according to the test conditions (two texture backgrounds $\times$ two texture locations $=$ four blocks). The test order of the four blocks was counterbalanced across observers. The test order of the 10 trials within each block (five target distances $\times$ two repeats) was fully randomized. The same test procedure was used as in Experiment 1.

\section{Results}

The average judged distance data are plotted in the graphs in Figure 6. A $2 \times 2 \times 5$ repeated measures ANOVA was used to analyze the data. The judged distances were larger in the convergence than in the parallel condition [main effect of texture background, $F(1,7)=12.751, p<.01$; background $\times$ distance, $F(4,28)=5.083, p<.005]$. Furthermore, a comparison between the texture locations in Figures 6A and 6B reveals a significant effect of texture location on judged distances [main effect of location, $F(1,7)=$ $8.549, p<.025$; location $\times$ distance, $F(4,28)=3.235, p<$ $.05]$. Overall, the fact that an effect of linear perspective can be revealed with just four elements in a dot-texture background suggests that the human visual system is remarkably efficient at processing linear perspective information.

\section{EXPERIMENT 5 Blind-Throwing Task}

Our experiments thus far have employed a verbal report task to measure observers' perceived distances. To demonstrate that the effect of linear perspective on perceived distance is task independent, in the present experiment we used a blind-throwing task, which is a visually directed action task, to measure judged distance (see, e.g., Eby \& Loomis, 1987; He et al., 2004).

\section{Method}

Observers. Six new observers participated in this experiment after providing informed consent. All of the observers were familiar with the task of throwing, since they frequently played basketball and/or volleyball (self-reported). They had normal or corrected-to-normal visual acuity and were naive to the purpose of the experiment.

Design. After a practice session in the blind-throwing task, we ran a baseline condition (full cue in the light) followed by a test condition (convergence and parallel conditions in the dark room). The baseline condition included six target distances $(2.5,3.75,5.0$, $6.25,7.5$, and $9.0 \mathrm{~m}$ ), and the test condition included three target distances $(3.75,5.0$, and $6.25 \mathrm{~m})$. The observers were tested twice at each distance. Trials in the test condition were blocked according to the texture patterns, but randomized within each block. The testing order of the two blocks was counterbalanced across observers.

Stimuli. The experimental settings in the dark room for the convergence and parallel test conditions in the present experiment (Figure 7) were similar to those in Experiment 3 (Figure 6B). Each condition had a line-texture background made up of two, instead of three, phosphorescent lines (each $2.54 \mathrm{~cm}$ wide and $1 \mathrm{~mm}$ thick) pasted on the level floor surface. The same dimly lit, red target from Experiment 1 was used as the test target. For the baseline condition, the experiment was conducted in a lighted environment on a level, carpeted floor surface. An orange hockey puck $(7.62 \mathrm{~cm}$ in diameter and $2.54 \mathrm{~cm}$ in height) served as the target.

Procedure. Immediately after Phase 1, the observer walked to another lighted hallway in the same building to begin testing in the baseline condition (Phase 2). The observer was blindfolded before each blind-throwing trial in order to allow the experimenter to set the target (the orange hockey puck) at a predetermined test distance on the level floor surface. Once the target was set, the experimenter instructed the observer to remove the blindfold to judge the target distance. To respond to the judged distance, the observer put on the blindfold again and threw the beanbag to the remembered target location. The experimenter looked for the initial landing location of the beanbag and marked the floor there. The experimenter then measured the distance between the marked location and the observer's feet, which was taken to be the judged target distance. The marker
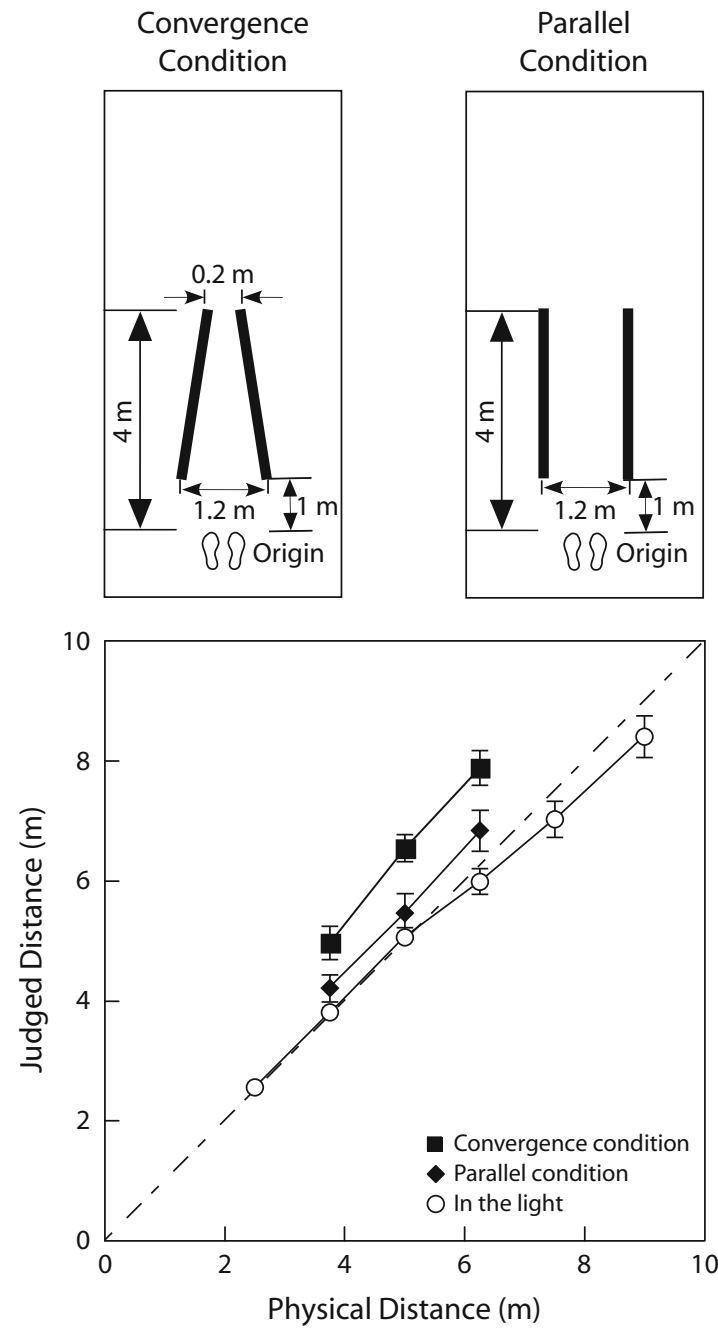

Figure 7. Experiment 5. (Top) Ground surface layouts in the convergence and parallel conditions, using line-texture displays. (Bottom) Judged target distance as a function of physical distance. Judged distances were larger in the convergence condition (squares) than in the parallel condition (diamonds). The open circles are the data from a control condition in a full-cue lighted environment. Unlike the other experiments reported in this article, in which a verbal report task was used, this experiment was conducted using a blind-throwing task. 
was then removed, to prevent it from serving as feedback for the observer. During this time, the observer remained blindfolded until the experimenter had finished preparing for the next trial.

For Phase 3 (the test condition), the observer performed the blindthrowing task in the same dark room used in Experiments 1-4. The blind-throwing task was the same as in Phase 2, but the observer waited in the lighted "transit room" before each trial while the experimenter placed the dimly lit target at the predetermined test distance. When the experimenter was ready, he led the observer, who had put on the blindfold while waiting, to the starting position in the dark room. Depending on the test condition (convergence or parallel), the appropriate phosphorescent line texture was visible on the floor. The experimenter then turned on the dimly lit target and informed the observer to temporarily remove the blindfold to judge the target distance. Once the observer had judged the target distance, he or she put on the blindfold again, instructed the experimenter to turn on a flashlight, and proceeded to throw the beanbag to the remembered target location. The experimenter located and marked the location at which the beanbag initially landed on the floor with the aid of the flashlight. By marking the response location in each trial, the experimenter was able to delay measuring the responded (thrown) distances until the experiment was completed, thus shortening the time that elapsed between trials.

The observers were not given any feedback regarding their performances in either Phase 2 or 3 of the experiment. Music from a radio cassette player was played aloud during the experiment to prevent the observers from using as feedback the acoustic cues associated with the beanbag being thrown and landing on the floor.

\section{Results}

The graph in Figure 7 plots the average data in the test and baseline conditions. The overestimation of the average distances was larger in the convergence condition than in the parallel condition, a finding consistent with our earlier results obtained using the verbal report task. A two-way repeated measures ANOVA revealed that the main effect of texture background was significant $[F(1,5)=65.099$, $p<.001]$, but the interaction effect was not $[F(2,10)=$ $2.636, p>.1]$.

The judged distances were larger in both the convergence and parallel conditions than in the baseline (fullcue) condition. A two-way repeated measures ANOVA of the data at the three test target distances (3.75, 5.0, and $6.25 \mathrm{~m}$ ) revealed that judged distances in the parallel condition were significantly larger than in the baseline condition [main effect, $F(1,5)=7.029, p<.05$; interaction, $F(2,10)=9.495, p<.01]$. (We will defer our discussion of the possible cause of larger-than-baseline judged distances in the convergence and parallel conditions until the General Discussion section.)

\section{EXPERIMENT 6 \\ Effect of Linear Perspective on the Judged Eye Level}

According to the prediction depicted in Figure 2, a converging line texture on a level floor surface should induce both a lower perceived eye level and a distance overestimation relative to a parallel line texture. In this experiment, we tested this prediction by measuring both judged eye level and judged distance in the convergence and parallel conditions. The same two-line texture backgrounds used in Experiment 5 (Figure 7) were employed in this experiment.
We further tested the prediction by adding two additional conditions, which essentially modified the backgrounds with a two-line texture to backgrounds with a one-line texture. Specifically, the left phosphorescent stripes in the convergence and parallel conditions were removed in order to create two new conditions. These were the parasagittal and oblique conditions, which were derived, respectively, from the parallel and convergence conditions. We reasoned that if linear perspective information (i.e., the relative orientation between two lines), rather than the orientation of a single line, played the critical role in distance judgments, we should expect to find little difference in performance between the parasagittal and oblique conditions, because a single line alone cannot provide linear perspective information.

\section{Method}

Observers. Six new observers participated in this experiment after providing informed consent. They were naive to the purpose of the study and had normal or corrected-to-normal visual acuity.

Design. For each of the four texture background conditions (parallel, convergence, parasagittal, and oblique), we measured observers' judged distances at five target distances $(1.5,2.5,3.75,5.0$, and $6.25 \mathrm{~m})$ and judged eye levels at four target distances $(2.5,3.75$, 5.0 , and $6.25 \mathrm{~m}$ ). Each texture background condition was tested in the same block. The order of testing target distances within a block was fully randomized. During the experiment, the observers were tested first in the eye-level judgment session, followed by the distance judgment session.

Stimuli. The convergence and parallel texture background conditions were the same as in Experiment 5 (Figure 7). In the parasagittal and oblique texture background conditions (not shown in Figure 8), only the right phosphorescent stripe from the parallel and convergence conditions, respectively, was presented; the left stripe was removed. The targets used for the eye-level judgment session were the same as the targets employed in the test condition in Experiment 1 (dimly lit red targets)

Procedure. For the eye-level session, we measured the observer's physical eye height before beginning the test session. During the test, the observer's judged eye level was measured in the dark room using two identical, dimly lit red targets. One target, which served as the reference light target, was placed on the level floor surface at one of the four target distances tested. The other was the test target; it was suspended directly above the reference target and could be adjusted vertically (up and down) by the experimenter (Figure 8A). In the trial, the observer fixated on the reference target on the floor and instructed the experimenter to move the test target vertically until it appeared at his or her eye level. The judged eye level was measured four times at each target distance. Following the eye-level judgment session, each observer's distance judgments were obtained using the verbal report task.

\section{Results and Discussion}

Figures $8 \mathrm{~B}$ and $8 \mathrm{C}$, respectively, show the average judged eye levels for the two-line and one-line texture background conditions. For the two-line texture background conditions, the average shifts in the judged eye level from the physical eye level were $-6.90^{\circ} \pm 2.40^{\circ}$ in the convergence condition and $-4.38^{\circ} \pm 1.99^{\circ}$ in the parallel condition (negative values indicate that the judged eye levels were lower than the physical eye level). These data were subjected to a two-way repeated measures ANOVA. The analysis revealed that the judged eye levels for the convergence condition were significantly 
lower than those for the parallel condition [mean difference, $\left.2.52^{\circ} ; F(1,5)=204.035, p<.001\right]$. This finding supports the prediction that converging lines cause the perceived eye level to shift downward. Our analysis also showed a significant main effect of target distance on judged eye level $[F(3,15)=12.726, p<.001]$. We believe this main effect of target distance is likely to be due to the observer's fixation position, because in a separate study at our laboratory (J. Wu et al., 2005) we found that the eye fixation position can affect the judged eye level. Recall that during the judged eye-level measurement in the present experiment, observers fixated their eyes on a reference target on the floor (immediately below the test target). Thus, the nearer the tested location, the lower an observer had to rotate his or her eye position downward. This then led to larger reductions in the judged eye level at near distances. Finally, an ANOVA fails to reveal a significant interaction effect between the texture backgrounds (convergence and parallel) and target distance $[F(3,15)=2.647, p>.08]$.

The average judged eye levels for the one-line texture background conditions are plotted in Figure 8C. A twoway repeated measures ANOVA failed to reveal a reliable difference between the parasagittal and oblique conditions $[F(1,5)=0.377, p>.5]$. This finding confirms our prediction that merely changing the orientation of a single line comprising the texture background does not affect the judged eye level. An ANOVA also showed that, as in the two-line texture background conditions, the judged eye levels in the one-line texture background conditions were significantly affected by the target distance $[F(3,15)=$ $15.740, p<.001]$. The analysis, however, failed to show a significant interaction effect between the line orientation and the target distance $[F(3,15)=2.451, p>.1]$.

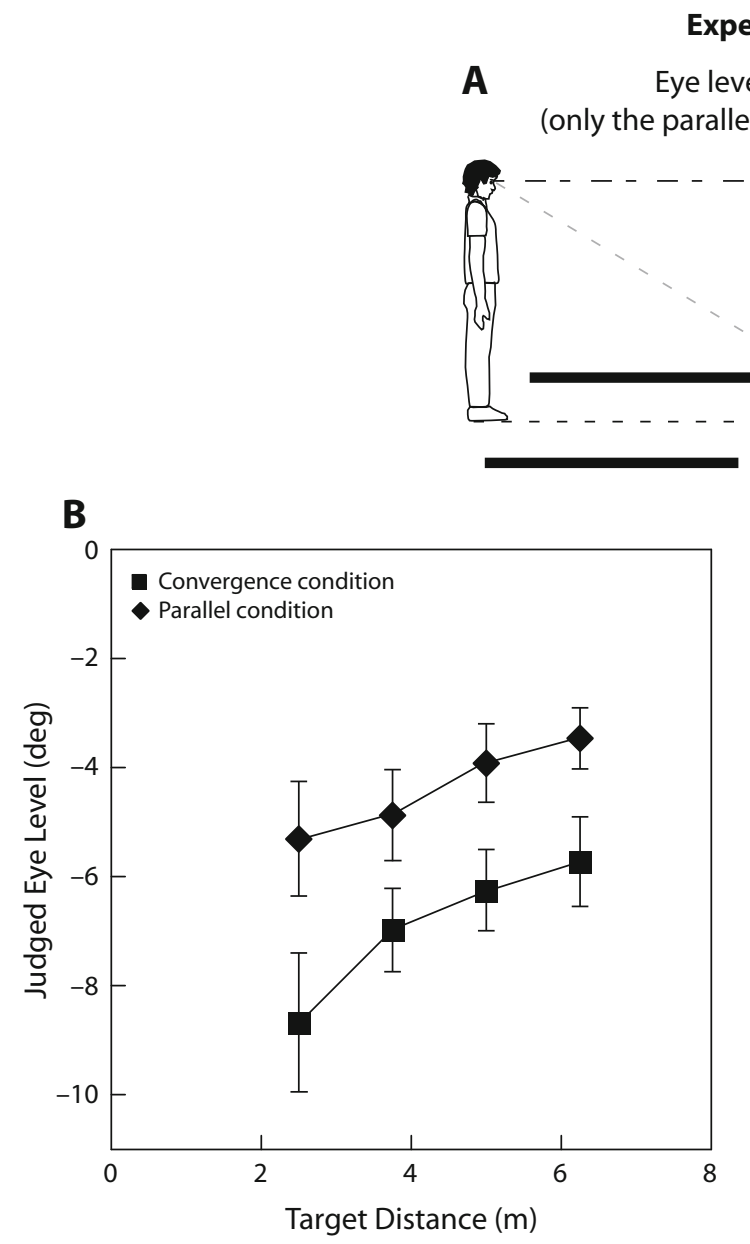

Experiment 6

e level judgment

(only the parallel condition is shown)
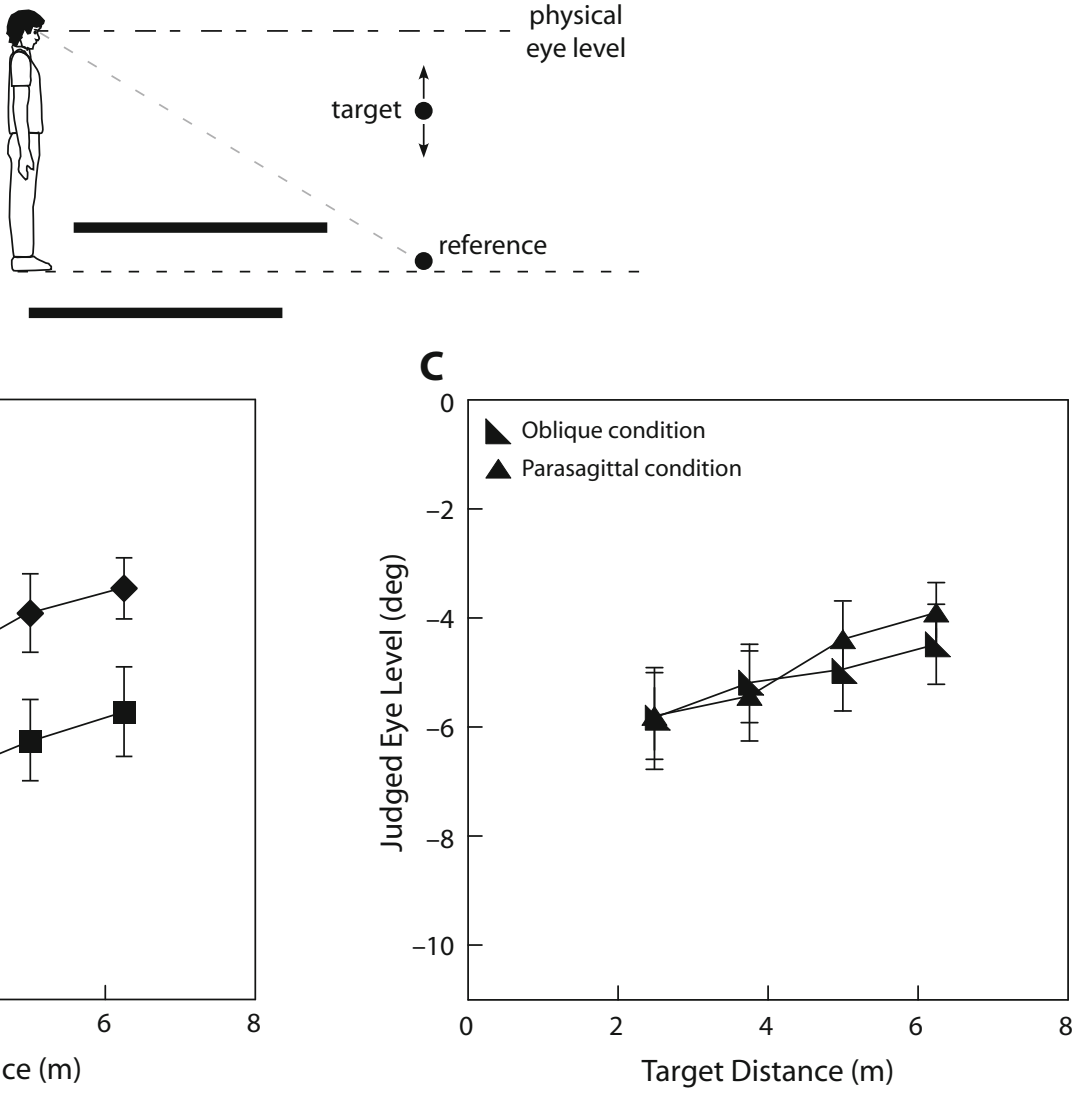

Figure 8. Experiment 6. (A) An illustration of the procedure for measuring judged eye level in the parallel condition. The observer fixated a reference target on the floor, while the experimenter moved up and down a test target positioned above the reference target, until the observer judged that the test target was at his or her eye level. Such eye-level measurements were performed at various distances by relocating the reference target to the desired viewing distance. Though it is not shown, the same eye-level judgment procedure was used for the other four conditions tested (convergence, dark, oblique, and parasagittal conditions). (B) Judged eye level as a function of target distance for the convergence (squares) and parallel (diamonds) conditions. Overall, judged eye levels were lower in the convergence than in the parallel condition. (C) Judged eye level as a function of target distance for the oblique (right triangles) and parasagittal (equilateral triangles) conditions. There was no significant difference between these two conditions. 
The average judged distances in the two-line and oneline texture background conditions are plotted in Figures 9A and $9 \mathrm{~B}$, respectively. Consistent with the prediction depicted in Figure 2B and the results of Experiments 2-5, observers overestimated distance relatively more in the convergence condition than in the parallel condition. This was confirmed by a two-way repeated measures ANOVA $[F(1,5)=13.746$, $p<.025]$. The analysis also showed that the relative amount of overestimation increased significantly with target distance $[F(4,20)=9.346, p<.001]$. In contrast, for the parasagittal and oblique conditions, the average judged distances were very similar between the two conditions [main effect of one-line texture background, $F(1,5)=0.001, p>.9$; background $\times$ distance, $F(3,15)=0.532, p>$.7]. This finding supports our hypothesis that the effect of texture background in the two-line conditions (Figure 9A) is associated with the linear perspective information provided by the lines, rather than the orientation of the lines per se.

Finally, we used the least-squares method to fit the mean judged distances in Figure 9 with curves based on the geometrical relationship $d=H / \tan (\alpha-\delta)$ described in Figure 2B. The curve fitting also allowed us to obtain the estimated shifts in judged eye levels. These were $-5.44^{\circ}$ and $-2.75^{\circ}$ for the convergence and parallel conditions, respectively. The difference between these derived eyelevel shifts is $2.69^{\circ}$, which is quite close to the difference obtained from the empirical measures of the judged eyelevel shift $\left(2.52^{\circ}\right)$. Using the same curve-fitting method, we also estimated the shifts in judged eye levels in the parasagittal and oblique conditions to be $-3.84^{\circ}$ and $-3.92^{\circ}$, respectively. The difference in shift between these two conditions is $0.09^{\circ}$, which is quite small.

Our findings also raise two issues that require further consideration. The first pertains to our data showing that the judged eye levels were similar in the oblique and parasagittal conditions (see Figure 8). No doubt, this finding is consistent with the notion that linear perspective information on the ground contributes to judged eye levels. However, it appears to be inconsistent with previous reports that the orientation of a single line that is pitched or placed in the frontoparallel plane can influence the judged eye level (e.g., Li \& Matin, 1996; Matin \& Li, 1994). We suspect this inconsistency could be due to the fact that the single lines in our experiment - unlike those studied by Matin and $\mathrm{Li}$-were placed on the ground surface. It is possible that the relative position of the single line with respect to an observer may influence its contribution to the judged eye level. It is also possible that since our observers had to make eye-level judgments while fixating on the reference target on the floor, the precision of their judgments may have been reduced. This relatively poor precision could have overwhelmed the response to a relatively small change in judged eye level caused by the orientation of a single line. In fact, we noticed that even with two lines in our parallel and convergence conditions, the effects of line orientation on the judged eye level (Figure 8) were smaller than the value (about $11^{\circ}$ ) predicted by the neural model proposed by Matin and Li (2001). Clearly, further experiments will be required in order to understand this apparent inconsistency.
The second issue pertains to our finding that the judged distances in the parasagittal and oblique conditions were similar to those in the parallel condition. This observation challenges the direct assumption that parallel lines, since they carry linear perspective information, should lead to larger judged distances than single lines, which do not carry linear perspective information. One likely explanation, similar to the one given in Experiment 2, is that the visual system also uses depth cues other than linear perspective to
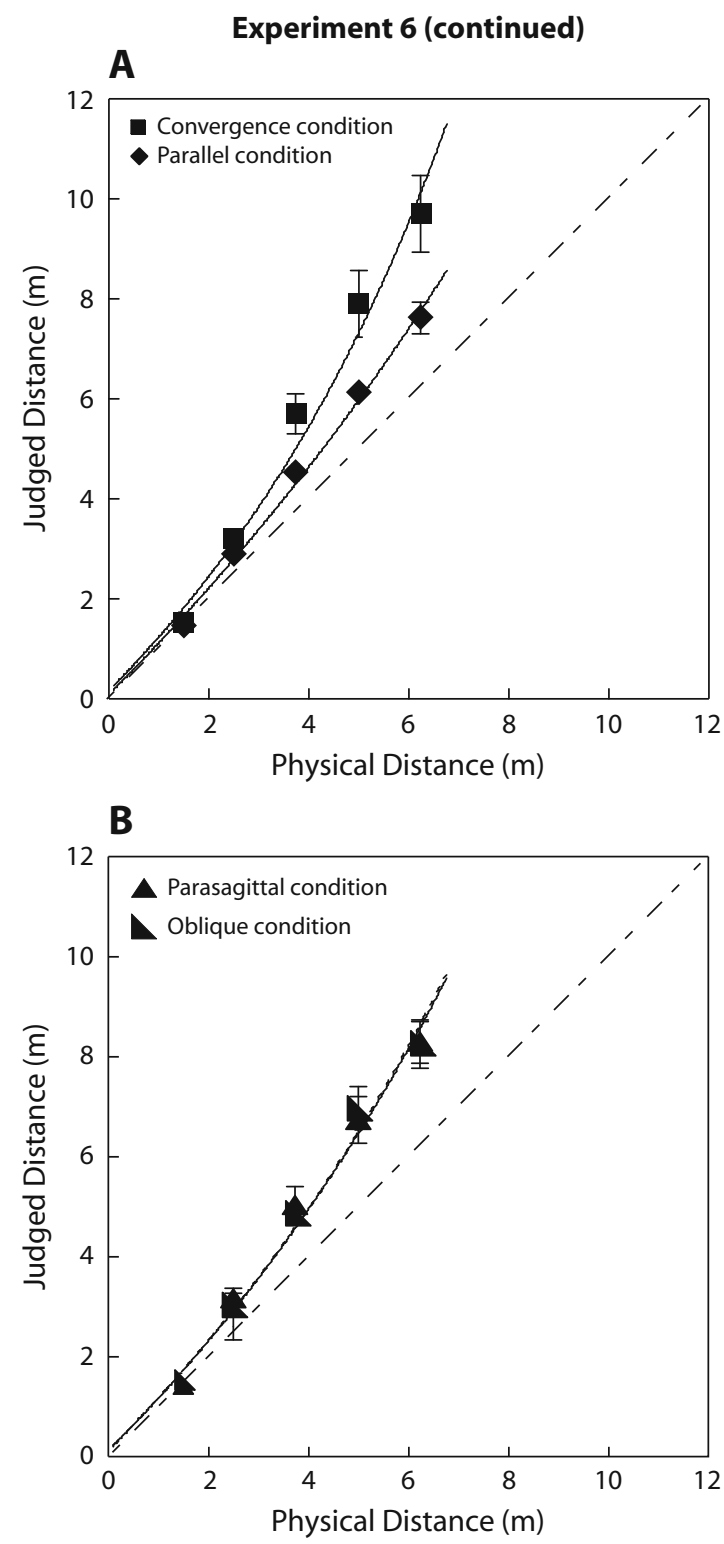

Figure 9. Experiment 6 (continued). After the measurements of eye level in each condition (see Figure 8), the observers were tested in a distance judgment experiment. (A and B) Judged distance as a function of physical distance. In panel $A$, judged distances in the convergence condition (squares) were larger than those in the parallel condition (diamonds). In panel B, there was no significant difference between the judged distances in the oblique condition (right triangles) and the parasagittal condition (equilateral triangles). 
construct the ground surface representation. Thus, for the single-line conditions (parasagittal and oblique), the visual system could rely on other depth cues, such as relative binocular disparity gradient and motion parallax, to form the ground surface representation. Adding a second line on the floor to one in the parasagittal or oblique condition (creating a parallel or convergence condition, respectively) means adding linear perspective. It is possible that the weighted contribution of the added linear perspective information to the ground surface representation is too modest to be observed in the distance judgment. This explanation predicts that reducing the contributions of other depth cues (e.g., binocular disparity and motion parallax) would lead to smaller judged distances with the single-line displays.

\section{EXPERIMENT 7 Linear Perspective Versus Compression Gradient}

Besides linear perspective information, texture compression gradient information on the ground surface can also be used for space perception (Gibson, 1950, 1979). Nevertheless, a number of studies in the past have shown that the visual system is more effective at deriving the orientation and depth of a surface from linear perspective than from compression gradient information (Andersen, Braunstein, \& Saidpour, 1998; Blessing et al., 1967; Braunstein \& Payne, 1969; Gillam, 1970). To verify whether this conclusion holds for the process of representing the ground surface in distance perception within a natural 3-D environment, the present experiment measured judged distances in two different texture background conditions (a compressing vs. an equal-spacing condition).

The left panels of Figures 10A and 10B are schematics of compressing and equal-spacing backgrounds in which dot texture and line texture, respectively, are used. It can be seen that the compression gradient information is stronger in the compressing texture background than in the equalspacing texture background condition. This comparison is akin to the one between the convergence and parallel texture background conditions utilizing linear perspective information. We predicted that if the visual system is as sensitive to compression gradient information as it is to linear perspective information, distance judgments would be overestimated more in the compressing condition than in the equal-spacing condition. The present experiment tested this prediction.

\footnotetext{
Method

Observers. Eight new observers participated in this experiment after providing informed consent. All had normal or corrected-to-normal visual acuity and were naive to the purpose of the experiment.

Design. The three independent variables tested were texture background (compressing vs. equal spacing), texture element type (line vs. dot texture), and target distance $(1.5,2.5,3.75,5.0$, and $6.25 \mathrm{~m}$ ). Each observer was tested in four blocks of trials (two texture backgrounds $\times$ two texture element types). The testing order of the four blocks was counterbalanced across observers. Each block comprised 10 trials ( 5 distances $\times 2$ repeats), and the test order of the trials within each block was randomized.

Stimuli. As illustrated in Figure 10, there were four stimulus conditions (two texture patterns $\times$ two texture element types). The texture element types were phosphorescent stripes to produce line texture
}

$(1.2 \mathrm{~m} \times 2.54 \mathrm{~cm}, 1 \mathrm{~mm}$ thick) and small, star-shaped phosphorescent elements to produce dot texture $(2.54 \times 2.54 \mathrm{~cm}, 1 \mathrm{~mm}$ thick $)$. These elements were pasted on the level floor surface of the dark room. To reduce the possible contribution of linear perspective information, neighboring rows of the dot or line elements were randomly misaligned with small left-right jitters. The same dimly lit, red light target used in Experiment 1 was used as the test target in this experiment.

Procedure. The same procedure was employed as in the test condition of Experiment 1.

\section{Results}

The mean judged distances are shown in the right panels of Figure 10. The data were subjected to a three-way repeated measures ANOVA. Overall, the analysis showed a significant effect of distance $[F(4,28)=82.49, p<$ $.001]$. However, it failed to reveal either a significant difference between the compressing and equal-spacing texture background conditions $[F(1,7)=0.035, p>.8]$ or a significant difference between the dot and line texture element types $[F(1,7)=0.480, p>.5]$. The analysis also failed to reveal any reliable interaction effects [texture background $\times$ distance, $F(4,28)=1.229, p>.3$; texture background $\times$ texture element type, $F(1,7)=0.115, p>$ .7; texture background $\times$ texture element type $\times$ distance, $F(4,28)=0.233, p>.9]$. These findings demonstrate that the visual system is not as sensitive to compression gradient information as to linear perspective information when representing a ground surface. Such insensitivity is consistent with the previous observations of others (Blessing et al., 1967; Braunstein \& Payne, 1969; Gillam, 1968, 1970). (It should be noted, however, that Andersen et al., 1998, using a different experimental setup, reported that compression gradient information was more effective than linear perspective information under certain conditions.)

Finally, even though we used a different group of observers in the present experiment, one might notice that the judged distances in the present experiment (Figure 10) were not smaller than those in the parallel condition of, say, Experiment 3 (Figure 5). After all, one might predict that with lateral jitters of the texture elements, the linear perspective information should be weakened, causing observers to see less depth. Thus, our findings in Figure 10 are somewhat surprising, though not entirely unexpected. We believe that, similar to the explanation offered for the results of Experiments 2 and 6, other depth cues besides linear perspective contributed to ground surface representation. Possibly the reduction in linear perspective information from the texture backgrounds of this experiment was not sufficient to cause a significant change in the ground surface representation and perceived distance.

\section{GENERAL DISCUSSION}

In this study, we examined the proposal that the visual system can use linear perspective information to construct perceptual space by applying two rules/assumptions: the parallel-line rule and the horizon rule. We measured both judged distances and eye levels in conditions in which a level floor surface was covered either by a converging or a parallel texture background in an otherwise dark room. Overall, our findings support the predictions of the pro- 

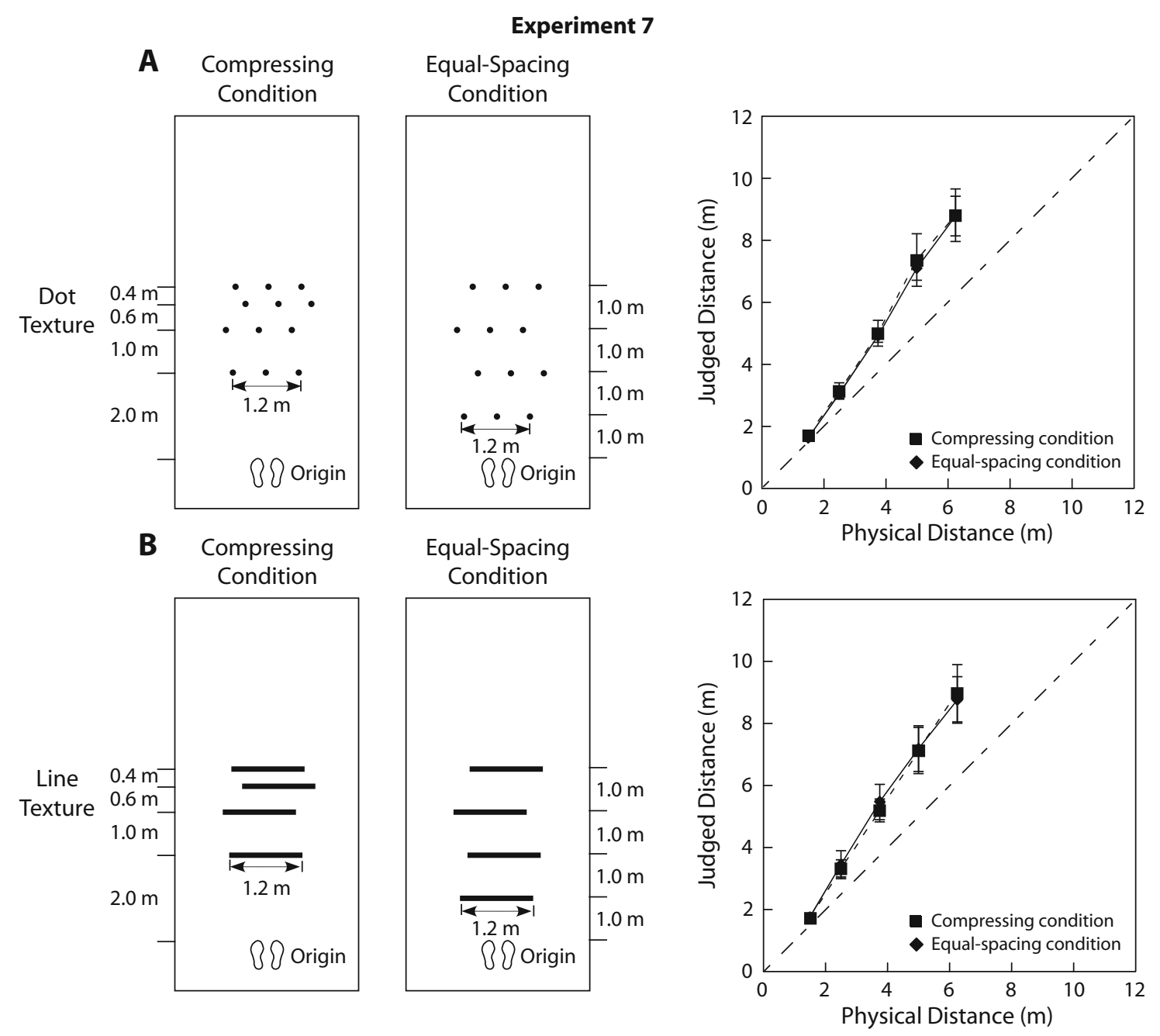

Figure 10. Experiment 7. (Left) Ground surface layouts in the compressing and equal-spacing conditions using dottexture (A) and line-texture (B) displays. (Right) The graphs in rows A and B plot judged target distance as a function of physical distance for the two texture background conditions. Overall, there was no significant difference between the judged distances in the compressing condition (squares) and the equal-spacing condition (diamonds).

posal (Figure 2) that distance is judged to be farther and eye level is judged to be lower with a converging than with a parallel texture background. Our observations also revealed that the human visual system is quite efficient at processing linear perspective information, because judged distance could be affected by linear perspective information contributed by as few as four phosphorescent elements on the floor (background). Furthermore, we found that, unlike with linear perspective information, manipulation of compression gradient information does not significantly impact distance judgments.

\section{A Possible Basis for Judged Distances in the Parallel Texture Condition}

Our data analyses and discussions have thus far concentrated mainly on the significant difference in judged distances between the convergence and parallel texture background conditions. Apart from this difference, we also found that judged distances were larger in the parallel texture background condition than in the full-cue condi- tion in all experiments except Experiment 1. For example, the judged distances (verbal report) in the parallel texture background condition of Experiment 2 (Figure 4) were larger than those in either the full-cue or the texture condition of Experiment 1 (Figure 3). A similar trend was observed in Experiment 5, which used a blind-throwing task (Figure 7). These results appear to be at odds with those of a different study from our laboratory (J. Wu et al., 2004).

J. Wu et al. (2004) used the blind-walking and gesturing task (Ooi et al., 2001) to measure the judged locations of dimly lit targets in conditions similar to those in the present study (complete darkness, as well as with a texture background on the level floor in an otherwise dark room). In the dark, a dimly lit target was perceived as being located at the intersection of the projection line from the eye to the target and an implicit slant surface (the visual system's intrinsic bias), a finding that is consistent with other studies from our laboratory (Ooi et al., 2001, 2006). Since the implicit surface was slanted with its far end upward from the level floor, a target in the intermedi- 
ate distance range was perceived as being nearer (i.e., the distance was underestimated) and higher than its actual physical location. J. Wu et al. (2004) also found, in parallel and convergence texture background conditions that were similar to those in the present study (see the left panels of Figures 11A and 11B, respectively), that the light targets were judged to be located along an implicit slant surface with a slant magnitude intermediate between the intrinsic bias and the level floor (the "ground representation" dashed lines in the right panels of Figure 11). The main difference in judgment between the convergence and parallel conditions was that a light target was judged to be higher and farther along the implicit slant surface in the former condition ("perceived target" in Figure 11). This finding suggests that the light target in the convergence condition was judged to have a smaller angular declination. In fact, this suggestion agrees with our finding in the present Experiment 6 of a lower judged eye level in the convergence condition (Figure 8). Yet, apart from this finding, we notice that the absolute magnitudes of judged distances in both the convergence and parallel conditions were underestimated in J. Wu et al. (2004), whereas they were overestimated in the present study. We believe this discrepancy is largely due to a critical procedural difference between the two studies.

This difference pertains to the instructions provided to the observers. The observers in the present study were explicitly told that the light target was always placed on the level floor surface, and that they had to judge the distance from their feet to the target on the floor (using either the verbal report or blind-throwing task). On the other hand, the observers in J. Wu et al.'s (2004) study were explicitly told that the light target could be placed either on the floor or at any elevation above the floor. To respond, they then had to walk blindfolded to traverse the horizontally judged distance between their body and the target and, upon reaching the destination, to indicate the height of the target using their hand (i.e., using a blind-walking and gesturing paradigm). So, how did such a procedural difference lead to larger judged distances in our present study? We believe that, in order to perform the verbal report or the blindthrowing task in the present study, the observers exploited their knowledge that the light target (as well as the texture background) was located on the level floor surface. Consequently, even when the light target was perceived above floor level (because of the influence of the intrinsic bias
A

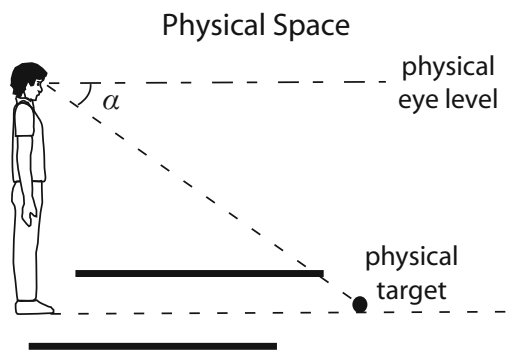

B

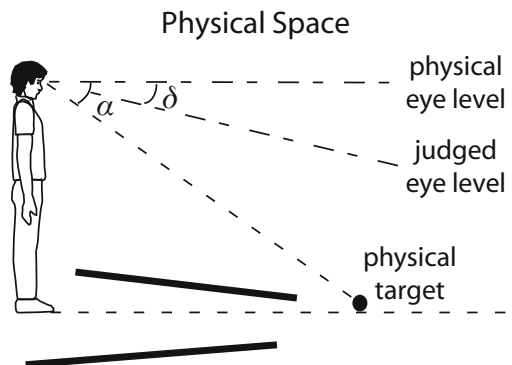

Parallel Condition

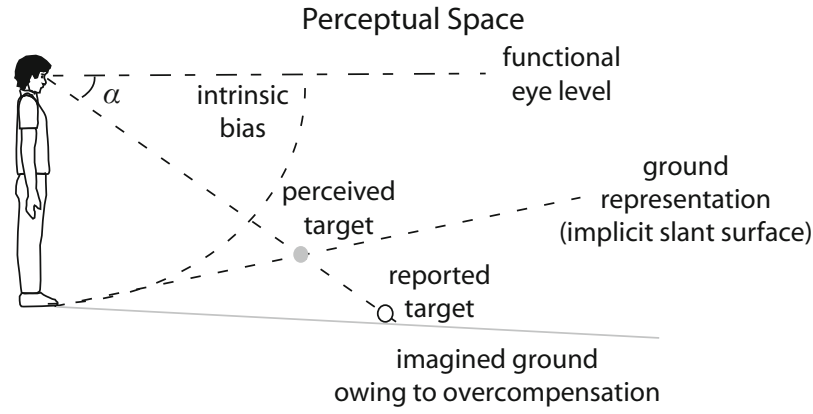

Convergence Condition

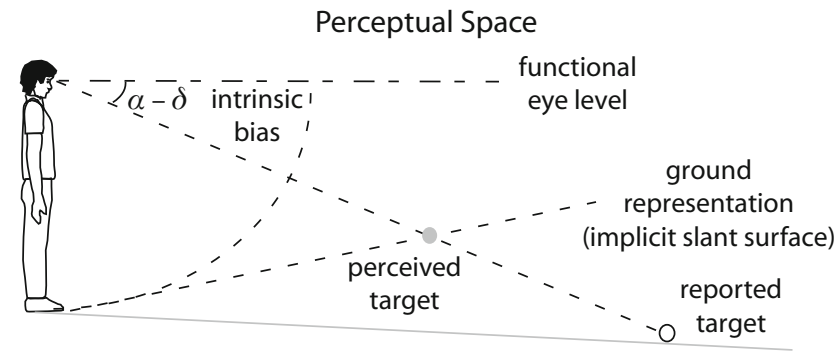

imagined ground

owing to overcompensation

Figure 11. A proposal of how distances were judged in the parallel (A) and convergence (B) conditions of our experiments. The left panels depict the experimental conditions in physical space, and the right panels depict the observers' perceptual spaces. Common to the perceptual space illustrations for both conditions are the intrinsic bias (dashed curves) and the lines for the ground representation (slanted dashed lines) and functional eye level (horizontal dashed lines parallel to the level floor). In each condition, a target is perceived at the point where the projection line from the eye to the target intersects the ground representation. The angular declination of the projection line to the target is determined in part by the perceived eye level, which is lowered in the convergence condition (J. Wu et al., 2004). The lowered eye level in this condition leads to the target being perceived as higher and farther away from the observer than in the parallel condition. In both conditions of the present study, though, the observers had explicit knowledge that the target was located on the floor, so they overcompensated for their perception by locating the target on an imagined ground surface (downward-slanting gray lines). 
in the dark), observers armed with the knowledge that the target was physically on the floor conceptually projected the target to the level floor surface (which was not visible in the dark). In other words, the observers intentionally compensated for the perceived height of the target by projecting the target below the level floor surface (see the "reported target" on the imagined ground surface in the right panels of Figure 11). This then led to the overestimation of target distance. As a further consequence, distance was even more overestimated in the convergence condition than in the parallel condition (cf. Figures 11A and 11B), because of the parallel-line rule.

Aside from the difference in instructions (i.e., in response criteria) as an explanation of why larger judged distances were found in the parallel condition than in the full-cue or the texture condition of Experiment 1, there are three other possible explanations. The first relates to our experimental design, in which the test blocks for the parallel and convergence conditions were counterbalanced across observers. It is possible that for the verbal report task, observers who performed the convergence block first might have been preconditioned by this block, and hence biased their responses accordingly when they performed the subsequent parallel block. Specifically, the reported distances in the subsequent parallel condition could have been scaled toward the distances in the convergence condition - that is, the observers could have responded with larger distances relative to those who performed the parallel block first. Therefore, when the final data from all of the parallel blocks were averaged, the judgments appeared to be overestimated. But since the main focus of our study was the difference between the parallel and convergence conditions, we do not have a sufficient sample size (i.e., number of observers) in each experiment to meaningfully assess this possibility through statistical analyses. Nevertheless, to explore that possibility, for all experiments we calculated average distances in the parallel blocks tested before and after the convergence blocks. These calculations did indeed reveal a tendency for judged distances in the parallel condition to be larger when they were tested after the convergence condition. Also, the judged distances for targets placed in the same locations were larger in the later parallel conditions than in the texture condition of Experiment 1. These two trends, though suggestive, are not conclusive, given our present sample size.

The second possible explanation is that, other than in Experiment 1, the texture backgrounds in our experiments extended on the floor in an area from 1 to $4 \mathrm{~m}$ from the observer (for an example, see Figure 4). In Experiment 1, though, the background in the texture condition extended from 1 to $9 \mathrm{~m}$. Thus, the far ground surface representation might not have been exactly the same across experiments; namely, the ground surface representation at a far distance was specified by the texture elements in Experiment 1, whereas in the remaining experiments it had to be extrapolated from the near texture surface representation. It is not clear whether this extrapolated far surface representation could have caused the overestimation of distances in the remaining experiments.
The third explanation relates to the visual system's tendency to treat the far terminals of elements, or the far edges of the texture background, as the horizon or eye level. As such, with nearer far terminals or edges (see, e.g., Figure 4), the eye level could have been shifted downward, resulting in decreased perceived angular declination and overestimation of distance.

\section{Perceived Eye Level, Angular Declination, and the Horizon Rule in Linear Perspective}

The angle subtended between the perceived eye level and the projection of an object constitutes the angular declination (i.e., height in the field) of the object below eye level. The angular declination of an object can be used by the visual system to compute the object's egocentric distance in the intermediate distance range (Gibson, 1950; Ooi et al., 2001; Philbeck \& Loomis, 1997). Given the significance of the angular declination, it is clear that the accuracy of the judged object distance depends also on the perceived eye level. This is because the perceived eye level serves as a reference for the vertical (up/down) direction.

The perceived eye level can be derived from both the body's senses (e.g., proprioceptive and vestibular) and external visual information (Cohen, 1973; Gibson, 1979; Matin \& Li, 1994, 1995; Post \& Welch, 1996; Sedgwick, 1973, 1986; Stoper \& Cohen, 1986, 1989). For example, our laboratory recently showed that optic flow information also influences the perceived eye level (J. Wu et al., 2005). When one walks on a level floor surface with a steady, straight-ahead eye fixation, the focus of expansion (FOE) of the optic flow coincides with the observer's eye level. J. Wu et al. (2005) revealed that the position of the FOE influences the perceived eye level. The study also showed an adaptation effect of the optic flow on the perceived eye level. With prolonged exposure to an optic flow display with vertically displaced FOE, the observer's perceived eye level after the removal of the optic flow stimulus was shifted in the direction of the displaced FOE. In addition, the adaptation of the perceived eye level led to a concordant change in judged target locations.

The relationship between texture gradient information and the horizon, which coincides with the perceived eye level when one stands on a level ground surface, has previously been explored. For example, Sedgwick $(1973,1986)$ proposed that the visual system can use horizon ratio information to judge the height of an object. He also pointed out that when the horizon is not seen in the visual environment, the visual system can derive the terrestrial horizon from perspective information on a level ground surface. To study the effectiveness of texture gradient information in distance perception, Andersen et al. (1998) had their observers verbally report the judged distances of stimuli on simulated texture scenes displayed on the computer screen. They found that judged distances were affected by the position of the vanishing point/vanishing line of the texture surfaces. Although Andersen et al. did not measure their observers' perceived eye levels, they hypothesized that their observers had possibly adopted the vanishing point/line as the horizon (hence, as eye level; Andersen et al., 1998). 
J. Wu et al. (2003) furnished evidence for the role of texture gradient (linear perspective) in eye-level and distance judgments in a real environment. They pasted two parallel lines on the walls to either side of the upright observer. The height of the midpoint of the lines was at the same level as the observer's eye height, and they varied the lines' orientation from the horizontal. They found that the orientation of the parallel lines affected both the judged eye level and the target egocentric distance. Our present study extends these observations by demonstrating that the orientation of lines (parallel and convergent) on the ground also significantly influences judged eye level and egocentric distance (see, e.g., Figures 9 and 10). In addition, the present study provides a geometrical foundation for the visual computation of linear perspective information on the basis of the horizon rule.

\section{Internal Perceptual Rules Used for Space Perception}

As mentioned in the introduction, we propose that the visual system uses an SSIP to construct the ground surface representation (He et al., 2004; B. Wu et al., 2004). According to this hypothesis, the representation of the ground surface is constructed from the weighted inputs of the visual system's intrinsic bias and the external depth information on the ground surface. In the extreme condition in which the ground surface is not visible (e.g., in the dark), the visual system relies on its intrinsic bias to localize objects. When the ground surface is visible, however, the visual system tends to rely more on the external depth information to represent the ground surface, and less on the intrinsic bias, except when the external information is inadequate. To accurately represent the ground surface using the external depth information, the SSIP first forms a representation of the near ground surface on the basis of the reliable near depth cues. The SSIP then uses the representation of the near ground surface as a template surface to sequentially integrate with farther patches of the ground surface based on the local texture gradient information of the far surface patches, to form a global ground surface representation.

How does the SSIP determine the slant of the ground surface on the basis of texture gradient information, though? Others have shown that the visual system can derive the optical slant of a local texture surface from its texture gradient information (e.g., Purdy, 1960; Sedgwick, $1983,1986)$. This computation is based on the visual system's assumptions about the structure of the texture on the ground surface (e.g., that texture elements on the ground have the same size). The experiments in the present study reveal that the visual system employs several assumptions or perceptual rules about the external surface to derive the surface slant from the local texture gradient information. For linear perspective information, a type of texture gradient information on the ground surface, the underlying assumptions are the parallel-line and horizon rules.

Our experiments further highlight a fundamental aspect of perceptual space in the intermediate distance range. Previous research has shown that when the ground surface is not visible, the visual system relies on its intrinsic bias to construct the perceptual space (e.g., Gogel \& Tietz, 1973;
Ooi et al., 2001, 2006). Our present experiments suggest that even when external depth information is available in a lighted environment, the visual system still relies on some assumptions (the internal perceptual rules) to form the perceptual space. The need to employ these perceptual rules may not be surprising, given that the optical information of the ground surface that impinges on the retina in a real-world environment is often not optimal (because of occlusion, texture discontinuation, and other factors), and is thus insufficient for constructing a veridical representation of the ground surface. In fact, it is a testament to the visual system's remarkable ability to utilize such perceptual rules to aid in its processing of complex optical information that our perceptual space appears veridical and our visually guided actions reasonably accurate.

\section{AUTHOR NOTE}

Parts of this work were presented at the 2001 Annual Meeting of the Vision Sciences Society (B. Wu, He, \& Ooi, 2001). B.W. is currently at the Department of Psychology, Carnegie Mellon University. We thank Myron Braunstein, Joe Lappin, and Hal Sedgwick for their constructive comments while reviewing an earlier draft of this article. This study was supported by Grant R01 EY014821 from the National Institutes of Health to Z.J.H. and T.L.O. Correspondence concerning this article should be addressed to either Z. J. He, Department of Psychological and Brain Sciences, University of Louisville, Louisville, KY 40292 (e-mail: zjhe@louisville.edu) or T. L. Ooi, Department of Basic Sciences, Pennsylvania College of Optometry, 8360 Old York Road, Elkins Park, PA 19027 (e-mail: tlooi@pco.edu).

\section{REFERENCES}

Andersen, G. J., Braunstein, M. L., \& Saidpour, A. (1998). The perception of depth and slant from texture in three-dimensional scenes. Perception, 27, 1087-1106.

Bian, Z., Braunstein, M. L., \& Andersen, G. J. (2005). The ground dominance effect in the perception of 3-D layout. Perception \& Psychophysics, 67, 802-815.

Blessing, W. W., Landauer, A. A., \& Coltheart, M. (1967). The effect of false perspective cues on distance and size judgments: An examination of the invariance hypotheses. American Journal of Psychology, 80, 250-256.

Braunstein, M. L., \& Payne, J. W. (1969). Perspective and form ratio as determinants of relative slant judgments. Journal of Experimental Psychology, 81, 584-590.

Bringoux, L., Tamura, K., Faldon, M., Gresty, M. A., \& Bronstein, A. M. (2004). Influence of whole-body pitch tilt and kinesthetic cues on the perceived gravity-referenced eye level. Experimental Brain Research, 155, 385-392.

CoHEN, M. M. (1973). Elevator illusion: Influences of otolith organ activity and neck proprioception. Perception \& Psychophysics, 14, 401-406.

Creem-Regehr, S. H., Willemsen, P., Gooch, A. A., \& Thompson, W. B. (2005). The influence of restricted viewing conditions on egocentric distance perception: Implications for real and virtual indoor environments. Perception, 34, 191-204.

Cutting, J. E., \& Millard, R. T. (1984). Three gradients and the perception of flat and curved surfaces. Journal of Experimental Psychology: General, 113, 198-216.

Cutting, J. E., \& Vishton, P. M. (1995). Perceiving layout and knowing distances: The integration, relative potency, and contextual use of different information about depth. In W. Epstein \& S. J. Rogers (Eds.), Perception of space and motion (Handbook of Perception and Cognition, 2nd ed., pp. 69-117). San Diego: Academic Press.

Eву, D. W., \& LoOMIs, J. M. (1987). A study of visually directed throwing in the presence of multiple distance cues. Perception \& Psychophysics, 41, 308-312.

ElliotT, D. (1987). Continuous visual information may be important after all: A failure to replicate Thomson (1983). Journal of Experimental Psychology: Human Perception \& Performance, 12, 388-391. 
Feria, C. S., Braunstein, M. L., \& Andersen, G. J. (2003). Judging distance across texture discontinuities. Perception, 32, 1423-1440.

GiBson, J. J. (1950). The perception of the visual world. Boston: Houghton Mifflin.

GiBson, J. J. (1979). The ecological approach to visual perception. Hillsdale, NJ: Erlbaum.

Gillam, B. J. (1968). Perception of slant when perspective and stereopsis conflict: Experiments with aniseikonic lenses. Journal of Experimental Psychology, 78, 299-305.

Gillam, B. [J.] (1970). Judgments of slant on the basis of foreshortening. Scandinavian Journal of Psychology, 11, 31-34.

Gogel, W. C., \& TietZ, J. D. (1973). Absolute motion parallax and the specific distance tendency. Perception \& Psychophysics, 13, 284-292.

HE, Z. J., \& OoI, T. L. (2000). Perceiving binocular depth with reference to a common surface. Perception, 29, 1313-1334.

He, Z. J., Wu, B., OoI, T. L., Yarbrough, G., \& Wu, J. (2004). Judging egocentric distance on the ground: Occlusion and surface integration. Perception, 33, 789-806.

Lappin, J. S., Shelton, A. L., \& Rieser, J. J. (2006). Environmental context influences visually perceived distance. Perception \& Psychophysics, 68, 571-581.

LI, W., \& MATIN, L. (1996). Visually perceived eye level is influenced identically by lines from erect and pitched planes. Perception, 25, 831-852.

Loomis, J. M., Da Silva, J. A., Fujita, N., \& Fukusima, S. S. (1992). Visual space perception and visually directed action. Journal of Experimental Psychology: Human Perception \& Performance, 18, 906-921.

Loomis, J. M., Da Silva, J. A., Philbeck, J. W., \& Fukusima, S. S. (1996). Visual perception of location and distance. Current Directions in Psychological Science, 5, 72-77.

Madison, C., Thompson, W., Kersten, D., Shirley, P., \& Smits, B. (2001). Use of interreflection and shadow for surface contact. Perception \& Psychophysics, 63, 187-194.

Matin, L., \& LI, W. (1994). Spatial summation among coextensive and parallel line segments across wide separations $\left(50^{\circ}\right)$ : Egocentric localization and the Great Circle Model. Vision Research, 34, 2577-2598.

Matin, L., \& LI, W. (1995). Multimodal basis for egocentric spatial localization and orientation. Journal of Vestibular Research, 5, 499-518.

Matin, L., \& LI, W. (2001). Neural model for processing the influence of visual orientation on visually perceived eye level (VPEL). Vision Research, 41, 2845-2872.

McCarley, J. S., \& He, Z. J. (2000). Asymmetry in 3-D perceptual organization: Ground-like surface superior to ceiling-like surface. Perception \& Psychophysics, 62, 540-549.

McCarley, J. S., \& He, Z. J. (2001). Sequential priming of 3-D perceptual organization. Perception \& Psychophysics, 63, 195-208.

Meng, J. C., \& Sedgwick, H. A. (2001). Distance perception mediated through nested contact relations among surfaces. Perception \& Psychophysics, 63, 1-15.

Meng, J. C., \& Sedgwick, H. A. (2002). Distance perception across spatial discontinuities. Perception \& Psychophysics, 64, 1-14.

Ni, R., Braunstein, M. L., \& Andersen, G. J. (2004). Perception of scene layout from optical contact, shadows and motion. Perception, 33, 1305-1318.

OoI, T. L., \& HE, Z. J. (2006). Localizing suspended objects in the intermediate distance range ( $>2$ meters) by observers with normal and abnormal binocular vision [Abstract]. Journal of Vision, 6(6), 422a. Retrieved June 2, 2007, from www.journalofvision.org/6/6/422/.

OoI, T. L., \& HE, Z. J. (2007). A distance judgment function based on space perception mechanisms: Revisiting Gilinsky's (1951) equation. Psychological Review, 114, 441-454.

OoI, T. L., Wu, B., \& He, Z. J. (2001). Distance determined by the angular declination below the horizon. Nature, 414, 197-200.

OoI, T. L., Wu, B., \& He, Z. J. (2006). Perceptual space in the dark affected by the intrinsic bias of the visual system. Perception, 35, 605-624.

Philbeck, J. W., \& Loomis, J. M. (1997). Comparison of two indicators of perceived egocentric distance under full-cue and reduced-cue conditions. Journal of Experimental Psychology: Human Perception \& Performance, 23, 72-85.

Post, R. B., \& Welch, R. B. (1996). The role of retinal versus perceived size in the effects of pitched displays on visually perceived eye level. Perception, 25, 853-859.

PURDY, W. C. (1960). The hypothesis of psychophysical correspondence in space perception (GE Tech. Info. Series, No. R60ELC56). Ithaca, NY: General Electric Advanced Electronics Center.

Rieser, J. J., Ashmead, D. H., Talor, C. R., \& Youngquist, G. A. (1990). Visual perception and the guidance of locomotion without vision to previously seen targets. Perception, 19, 675-689.

Rosinski, R. R., \& LEVINE, N. P. (1976). Texture gradient effectiveness in the perception of surface slant. Journal of Experimental Child Psychology, 22, 261-271.

Sauer, C. W., Braunstein, M. L., Andersen, G. J., \& Bian, Z. (2001). Judged shape of ground plane regions in realistic 3-D scenes [Abstract]. Journal of Vision, 1(3), 41a. Retrieved Oct. 2, 2006, from www.journalofvision.org/1/3/41/.

SEDGWICK, H. A. (1973). The visible horizon: A potential source of visual information for the perception of size and distance. Unpublished doctoral dissertation, Cornell University, Ithaca, NY.

SEDGWICK, H. A. (1983). Environment-centered representation of spatial layout: Available visual information from texture and perspective In J. Beck, B. Hope, \& A. Rosenfeld (Eds.), Human and machine vision (pp. 425-458). New York: Academic Press.

SEDGWICK, H. A. (1986). Space perception. In K. R. Boff, L. Kaufman, \& J. P. Thomas (Eds.), Handbook of perception and human performance: Vol. 1. Sensory processes and perception (pp. 21.1-21.57). New York: Wiley.

SinAI, M. J., OoI, T. L., \& He, Z. J. (1998). Terrain influences the accurate judgement of distance. Nature, 395, 497-500.

Stoper, A. E., \& Cohen, M. M. (1986). Judgments of eye level in light and in darkness. Perception \& Psychophysics, 40, 311-316.

Stoper, A. E., \& Cohen, M. M. (1989). Effect of structured visual environments on apparent eye level. Perception \& Psychophysics, 46, 469-475.

Thomson, J. A. (1983). Is continuous visual monitoring necessary in visually guided locomotion? Journal of Experimental Psychology: Human Perception \& Performance, 9, 427-443.

Vogel, J. M. \& Teghtsoonian, M. (1972). The effects of perspective alterations on apparent size and distance scales. Perception \& Psychophysics, 11, 294-298.

VRedeman de Vries, H. (1968). Perspective. New York: Dover. (Original work published 1604)

Wu, B. (2004). The visual perception of distance in action space. Unpublished doctoral dissertation, University of Louisville, Louisville, KY.

Wu, B., He, Z. J., \& OoI, T. L. (2001). Judging absolute distance by relying on linear perspective and texture density cues [Abstract]. Journal of Vision, 1(3), 383a. Retrieved Oct. 2, 2006, from www.journalofvision .org $/ 1 / 3 / 383 /$.

Wu, B., He, Z. J., \& OoI, T. L. (2007). Inaccurate representation of the ground surface beyond a texture boundary. Perception, 36, 703-721.

Wu, B., OoI, T. L., \& HE, Z. J. (2004). Perceiving distance accurately by a directional process of integrating ground information. Nature, 428, 73-77.

Wu, J., He, Z. J., \& OoI, T. L. (2003). Vertical and horizontal references determined by linear perspective and optic flow information [Abstract]. Journal of Vision, 3(9), 499a. Retrieved Oct. 2, 2006, from www.journalofvision.org/3/9/499/.

Wu, J., He, Z. J., \& OoI, T. L. (2004). Stimulus duration and binocular disparity factors in representing the ground surface and localizing object in the intermediate distance range [Abstract]. Journal of Vision, 4(8), 21a. Retrieved Oct. 2, 2006, from www.journalofvision .org $/ 4 / 8 / 21 /$.

Wu, J., HE, Z. J., \& OoI, T. L. (2005). Visually perceived eye level and horizontal midline of the body trunk influenced by optic flow. Perception, 34, 1045-1060.

Wu, J., He, Z. J., \& OoI, T. L. (2006). The slant of the visual system's intrinsic bias in space perception and its contribution to ground surface representation [Abstract]. Journal of Vision, 6(6), 730a. Retrieved Oct. 2, 2006, from www.journalofvision.org/6/6/730/

(Manuscript received April 18, 2006; revision accepted for publication September 29, 2006.) 